\title{
Octupole deformation in light actinides within an analytic quadrupole octupole axially symmetric model with Davidson potential
}

\author{
Dennis Bonatsos ${ }^{1}$, Andriana Martinou ${ }^{1}$, N. Minkov $^{2}$, S. Karampagia ${ }^{1}$, and D. Petrellis ${ }^{1,3}$ \\ ${ }^{1}$ Institute of Nuclear and Particle Physics, National Centre for Scientific \\ Research "Demokritos", GR-15310 Aghia Paraskevi, Attiki, Greece \\ ${ }^{2}$ Institute of Nuclear Research and Nuclear Energy, \\ Bulgarian Academy of Sciences, 72 Tzarigrad Road, 1784 Sofia, Bulgaria and \\ 3 Department of Physics, University of Istanbul, 34134 Vezneciler, Istanbul, Turkey
}

\begin{abstract}
The analytic quadrupole octupole axially symmetric model, which had successfully predicted ${ }^{226} \mathrm{Ra}$ and ${ }^{226} \mathrm{Th}$ as lying at the border between the regions of octupole deformation and octupole vibrations in the light actinides using an infinite well potential (AQOA-IW), is made applicable to a wider region of nuclei exhibiting octupole deformation, through the use of a Davidson potential, $\beta^{2}+$ $\beta_{0}^{4} / \beta^{2}$ (AQOA-D). Analytic expressions for energy spectra and B(E1), B(E2), B(E3) transition rates are derived. The spectra of ${ }^{222-226} \mathrm{Ra}$ and ${ }^{224,226} \mathrm{Th}$ are described in terms of the two parameters $\phi_{0}$ (expressing the relative amount of octupole vs. quadrupole deformation) and $\beta_{0}$ (the position of the minimum of the Davidson potential), while the recently determined B(EL) transition rates of ${ }^{224} \mathrm{Ra}$, presenting stable octupole deformation, are successfully reproduced. A procedure for gradually determining the parameters appearing in the $\mathrm{B}(\mathrm{EL})$ transitions from a minimum set of data, thus increasing the predictive power of the model, is outlined.

PACS numbers: 21.60.Ev, 21.60.Fw, 21.10.Re, 23.20.Js
\end{abstract}

\section{INTRODUCTION}

Rotational nuclear spectra have long been attributed to quadrupole deformations [1]. However, octupole deformations [corresponding to reflection asymmetric (pearlike) shapes] [2 4] are supposed to occur in certain regions, most notably in the light actinides [5] 8 and in some light rare earths 911. The hallmark of octupole deformation is a negative parity band with levels $L^{\pi}=1^{-}, 3^{-}, 5^{-}, \ldots$, lying close to the ground state band and forming with it a single band with $L^{\pi}=0^{+}$, $1^{-}, 2^{+}, 3^{-}, 4^{+}, \ldots$, while a negative parity band lying systematically higher than the ground state band is a footprint of octupole vibrations.

The transition from the regime of octupole vibrations into the region of octupole deformation has been considered by several authors [12 14. In the analytic quadrupole octupole axially symmetric (AQOA) model [15], the actinides lying on the border between the regions of octupole deformation and octupole vibrations have been described, making the following assumptions.

1) Quadrupole and octupole deformations are taken into account on equal footing, their relative presence described by the only free parameter in the model, $\phi_{0}$.

2) Axial symmetry is assumed, in order to keep the problem tractable.

3) Separation of variables is achieved in a way analogous to the one used in the framework of the $\mathrm{X}(5)$ model [16, describing the first order shape phase transition between spherical and quadrupole deformed shapes [17.

4) An infinite well potential is assumed appropriate for the description of the border region, as in the $\mathrm{E}(5)$ [18] and $\mathrm{X}(5)$ [16 models, the former one describing the second order shape phase transition between spherical and $\gamma$-unstable nuclei. Therefore we are going to call this solution the AQOA-IW model.

A different approach to the problem of phase transition in the octupole mode has been developed by Bizzeti and Bizzeti-Sona [19, 20], characterized by the introduction of a new parametrization of the quadrupole and octupole degrees of freedom, using as intrinsic frame of reference the principal axes of the overall tensor of inertia, as resulting from the combined quadrupole and octupole deformation. The main differences between the two models are:

1) The AQOA model is analytic, while the model of Refs. [19, 20] is not.

2) In the AQOA model the symmetry axes of the quadrupole and octupole deformations are taken to coincide, in order to guarantee axial symmetry, while in the more general framework of Refs. [19, 20] nonaxial contributions, small but not frozen to zero, are taken into account.

In both models [15, 19, 20, ${ }^{226} \mathrm{Ra}$ and ${ }^{226} \mathrm{Th}$ appear to lie close to the point of transition between octupole deformation and octupole vibrations, with heavier isotopes corresponding to octupole vibrations and lighter isotopes exhibiting octupole deformation.

The recent experimental verification of stable octupole deformation in ${ }^{224} \mathrm{Ra}$ [21] stirred interest in octupole deformation in the light actinides and their theoretical interpretation. The AQOA model can be made applicable to deformed nuclei near the transition point by replacing the infinite well potential by the Davidson potential 22] of the form $\beta^{2}+\beta_{0}^{4} / \beta^{2}$, which contains an additional free parameter, the position $\beta_{0}$ of the minimum of the potential well. The flexibility acquired through the replacement of the infinite well potential by the Davidson potential has been demonstrated and exploited in the case of quadrupole deformation in [23. The analytic quadrupole 
octupole axially symmetric model with a Davidson potential, to be called the AQOA-D model, is the subject of the present work. In addition to the spectra of ${ }^{222-226} \mathrm{Ra}$ [7, 8] and ${ }^{224,226} \mathrm{Th}$ [24, 25], the recently measured [21] electric transition probabilities of ${ }^{224} \mathrm{Ra}$ provide an excellent test ground for the model, already exploited in the Bizzeti and Bizzeti-Sona approach [26].

The above mentioned work on the octupole degree of freedom has been developed in the framework of the collective model [1. Alternative approaches include the following.

1) A complete algebraic classification of the states occurring in the simultaneous presence of the quadrupole and octupole degrees of freedom has been provided in terms of the spdf-interacting boson model [27 30], which has been successfully applied to Ra 31], Th [31, U [32], and $\mathrm{Pu} 32$ isotopes. Mean field studies of the critical point for the onset of octupole deformation in quadrupole deformed systems have been carried out in Refs. [33, 34].

2) An alternative interpretation of the low-lying negative parity states in the light actinides has been provided in terms of clustering [35 39]. The recent experimental findings for ${ }^{224} \mathrm{Ra}$ [21] seem to point against this interpretation, but wider evidence in more nuclei is desirable.

3) Relativistic mean field calculations involving the octupole degree of freedom have been carried out both in the light actinides region [40, 41, and in the light rare earths [42, 43], corroborating [41, 45, the transition from octupole deformation to octupole vibrations in the light Th isotopes.

4) A hybrid approach combining the algebraic approach of the interacting boson model of 1) with the relativistic energy density functional theory of 3) has been recently developed 44 and applied the $\mathrm{Ra}$ and Th isotopes [45, 46], as well as to the rare earths $\mathrm{Ba}$ and $\mathrm{Sm}$ [46], again corroborating the transition from octupole deformation to octupole vibrations in the light $\mathrm{Ra}$ and $\mathrm{Th}$ isotopes.

5) The extended coherent state model (ECSM) has been successfully applied to the description of several negative parity bands in $\mathrm{Rn}$ [47, Ra 47 50, Th [50, $\mathrm{U}$ [50], and $\mathrm{Pu}$ [50] isotopes.

The AQOA-D model is described in Section II, while in Section III numerical results are provided and subsequently discussed in Section IV. The integrals needed in the calculation of electric transition probabilities are calculated in Appendices A-C, while in Appendix D some details of the derivation of the Hamiltonian, the method of solution and the comparison to other approaches are given.

\section{THE ANALYTIC QUADRUPOLE OCTUPOLE AXIALLY SYMMETRIC (AQOA) MODEL}

\section{A. Formulation}

In the AQOA model [15] the following assumptions are made:

a) The axes of the quadrupole and octupole deformations are taken to coincide. In other words, axial symmetry is assumed, while the $\gamma$ degree of freedom is ignored.

b) Levels with $K \neq 0$ (where $K$ is the projection of the angular momentum on the body-fixed $z^{\prime}$ axis) are ignored, since they are lying infinitely high in energy [51].

The Hamiltonian of the AQOA model reads [51, 52]

$$
\begin{array}{r}
H=-\sum_{\lambda=2,3} \frac{\hbar^{2}}{2 B_{\lambda}} \frac{1}{\beta_{\lambda}^{3}} \frac{\partial}{\partial \beta_{\lambda}} \beta_{\lambda}^{3} \frac{\partial}{\partial \beta_{\lambda}}+\frac{\hbar^{2} \hat{L^{2}}}{6\left(B_{2} \beta_{2}^{2}+2 B_{3} \beta_{3}^{2}\right)} \\
+V\left(\beta_{2}, \beta_{3}\right)
\end{array}
$$

where $\beta_{2}$ and $\beta_{3}$ are the quadrupole and octupole deformations, $B_{2}, B_{3}$ are the mass parameters, and $\hat{L}$ is the angular momentum operator in the intrinsic frame, taken along the principal axes of inertia.

The solutions of the Schrödinger equation read [51]

$$
\Phi_{L}^{ \pm}\left(\beta_{2}, \beta_{3}, \theta\right)=\left(\beta_{2} \beta_{3}\right)^{-3 / 2} \Psi_{L}^{ \pm}\left(\beta_{2}, \beta_{3}\right)|L M 0, \pm\rangle
$$

where $\theta$ are the Euler angles describing the orientation of the body-fixed axes $x^{\prime}, y^{\prime}, z^{\prime}$ relative to the laboratoryfixed axes $x, y, z$, while the function $|L M 0, \pm\rangle$ describes the rotation of an axially symmetric nucleus with angular momentum projection $M$ onto the laboratory-fixed $z$-axis and projection $K=0$ onto the body-fixed $z^{\prime}$-axis [1]

$$
|L M 0, \pm\rangle=\sqrt{\frac{2 L+1}{32 \pi^{2}}}\left(1 \pm(-1)^{L}\right) \mathcal{D}_{0, M}^{L}(\theta),
$$

with $\mathcal{D}(\theta)$ denoting Wigner functions of the Euler angles.

Wave functions with the + label correspond to positive parity states with $L=0,2,4, \ldots$, while these with the - label correspond to negative parity states with $L=1$, $3,5, \ldots$.

The Schrödinger equation can be simplified by introducing 51, 52]

$$
\tilde{\beta}_{2}=\beta_{2} \sqrt{\frac{B_{2}}{B}}, \quad \tilde{\beta}_{3}=\beta_{3} \sqrt{\frac{B_{3}}{B}}, \quad B=\frac{B_{2}+B_{3}}{2},
$$

reduced energies $\epsilon=\left(2 B / \hbar^{2}\right) E$ and reduced potentials $v=\left(2 B / \hbar^{2}\right) V$, as well as polar coordinates (with $0 \leq$ $\tilde{\beta}<\infty$ and $-\pi / 2 \leq \phi \leq \pi / 2$ ) [51, 52]

$$
\tilde{\beta}_{2}=\tilde{\beta} \cos \phi, \quad \tilde{\beta}_{3}=\tilde{\beta} \sin \phi, \quad \tilde{\beta}=\sqrt{\tilde{\beta}_{2}^{2}+\tilde{\beta}_{3}^{2}},
$$


leading to

$$
\begin{aligned}
& {\left[-\frac{\partial^{2}}{\partial \tilde{\beta}^{2}}-\frac{1}{\tilde{\beta}} \frac{\partial}{\partial \tilde{\beta}}+\frac{L(L+1)}{3 \tilde{\beta}^{2}\left(1+\sin ^{2} \phi\right)}-\frac{1}{\tilde{\beta}^{2}} \frac{\partial^{2}}{\partial \phi^{2}}\right.} \\
& \left.+v(\tilde{\beta}, \phi)+\frac{3}{\tilde{\beta}^{2} \sin ^{2} 2 \phi}-\epsilon_{L}\right] \Psi_{L}^{ \pm}(\tilde{\beta}, \phi)=0 .
\end{aligned}
$$

In addition, separation of variables can be achieved by assuming the potential to be of the form $v(\tilde{\beta}, \phi)=$ $u(\tilde{\beta})+w\left(\tilde{\phi}^{ \pm}\right)$, where $w\left(\tilde{\phi}^{ \pm}\right)$is supposed to be of the form of two very steep harmonic oscillators centered at the values $\pm \phi_{0}$. In this way Eq. (6) is separated into

$$
\begin{array}{r}
{\left[-\frac{\partial^{2}}{\partial \tilde{\beta}^{2}}-\frac{1}{\tilde{\beta}} \frac{\partial}{\partial \tilde{\beta}}+\frac{1}{\tilde{\beta}^{2}}\left(\frac{L(L+1)}{3\left(1+\sin ^{2} \phi_{0}\right)}+\frac{3}{\sin ^{2} 2 \phi_{0}}\right)\right.} \\
\left.+u(\tilde{\beta})-\epsilon_{\tilde{\beta}}(L)\right] \psi_{L}(\tilde{\beta})=0,
\end{array}
$$

and

$$
\left[-\frac{1}{\left\langle\tilde{\beta}^{2}\right\rangle} \frac{\partial^{2}}{\partial\left(\tilde{\phi}^{ \pm}\right)^{2}}+w\left(\tilde{\phi}^{ \pm}\right)-\epsilon_{\phi}\right] \chi\left(\tilde{\phi}^{ \pm}\right)=0
$$

where $\Psi_{L}^{ \pm}(\tilde{\beta}, \phi)=N_{\tilde{\beta}} \psi_{L}(\tilde{\beta}) N_{\phi}\left(\chi\left(\tilde{\phi}^{+}\right) \pm \chi\left(\tilde{\phi}^{-}\right)\right) / \sqrt{2}$, with $N_{\tilde{\beta}}$ and $N_{\phi}$ being normalization factors, while $\left\langle\tilde{\beta}^{2}\right\rangle$ is the average of $\tilde{\beta}^{2}$ over $\psi(\tilde{\beta})$, and $\epsilon_{L}=\epsilon_{\tilde{\beta}}(L)+\epsilon_{\phi}$.

On the above the following comments apply:

a) $\phi=0$ corresponds to quadrupole deformation alone, while $\phi= \pm \pi / 2$ corresponds to octupole deformation alone.

b) Because of the two steep oscillators involved, $\phi$ remains close to $\pm \phi_{0}$ and, therefore, the relative amount of quadrupole and octupole deformation remains constant.

Some details of the derivation of the Hamiltonian, the method of solution, and the comparison of the present model to other approaches are given in Appendices D1D4.

\section{B. The $\tilde{\beta}$ part of the spectrum}

Eq. (7) is exactly soluble [53, 54] in the case of the Davidson potentials 22

$$
u(\tilde{\beta})=\tilde{\beta}^{2}+\frac{\tilde{\beta}_{0}^{4}}{\tilde{\beta}^{2}},
$$

in which the eigenfunctions are Laguerre polynomials

$$
F_{n_{\beta}}^{L}(\tilde{\beta})=\sqrt{\frac{2 n_{\beta} !}{\Gamma\left(n_{\beta}+a_{L}+1\right)}} \tilde{\beta}^{a_{L}} L_{n_{\beta}}^{a_{L}}\left(\tilde{\beta}^{2}\right) e^{-\tilde{\beta}^{2} / 2},
$$

with

$$
a_{L}=\sqrt{\frac{L(L+1)}{3\left(1+\sin ^{2} \phi_{0}\right)}+\frac{3}{\sin ^{2} 2 \phi_{0}}+\beta_{0}^{4}}
$$

while the energy eigenvalues are given by

$$
\begin{aligned}
E_{n_{\beta}, L}=2 n_{\beta} & +a_{L}+1=2 n_{\beta}+1 \\
& +\sqrt{\frac{L(L+1)}{3\left(1+\sin ^{2} \phi_{0}\right)}+\frac{3}{\sin ^{2} 2 \phi_{0}}+\beta_{0}^{4}} .
\end{aligned}
$$

Eq. (7) is also exactly soluble in the case of an infinite well potential in $\tilde{\beta}$, in which the eigenfunctions are Bessel functions. This solution has been worked out in Ref. [15.

\section{The $\phi$ part of the spectrum}

Eq. (8) with the potential corresponding to two harmonic oscillators centered at $\pm \phi_{0}$

$$
w\left(\tilde{\phi}^{ \pm}\right)=\frac{1}{2} c\left(\phi \mp \phi_{0}\right)^{2}=\frac{1}{2} c\left(\tilde{\phi}^{ \pm}\right)^{2}, \quad \tilde{\phi}^{ \pm}=\phi \mp \phi_{0},
$$

has been solved in Ref. 15. The energy eigenvalues are

$$
\epsilon_{\phi}=\sqrt{\frac{2 c}{\left\langle\tilde{\beta}^{2}\right\rangle}}\left(n_{\phi}+\frac{1}{2}\right), \quad n_{\phi}=0,1,2, \ldots
$$

where $n_{\phi}$ is the number of quanta in the $\phi$ degree of freedom, while the eigenfunctions are Hermite polynomials $H_{n_{\phi}}$

$$
\begin{aligned}
\chi_{n_{\phi}}\left(\tilde{\phi}^{ \pm}\right)=N_{n_{\phi}} H_{n_{\phi}}\left(b \tilde{\phi}^{ \pm}\right) e^{-b^{2}\left(\tilde{\phi}^{ \pm}\right)^{2} / 2} & \\
b & =\left(\frac{c\left\langle\tilde{\beta}^{2}\right\rangle}{2}\right)^{1 / 4},
\end{aligned}
$$

with normalization constant $N_{n_{\phi}}=\sqrt{\frac{b}{\sqrt{\pi} 2^{n_{\phi}} n_{\phi} !}}$.

The total energy in the present model is then

$$
\begin{aligned}
E\left(n_{\beta}, L, \phi_{0}, n_{\phi}\right) & =E_{0}+C_{1} E_{n_{\beta}, L}+C_{2} n_{\phi}, \\
C_{1} & =\frac{\hbar^{2}}{2 B}, \quad C_{2}=\frac{\hbar^{2}}{2 B} \sqrt{\frac{2 c}{\left\langle\beta^{2}\right\rangle}} .
\end{aligned}
$$

In what follows only bands with $n_{\phi}=0$ will be considered.

As an example of the dependence of the energy levels on the free parameters $\beta_{0}$ and $\phi_{0}$ in Eq. (12), the energy levels with $n_{\beta}=0$ and $L=2$ are shown in Fig. 1 . Smooth variation with both parameters is seen. It is worth remarking that very slight dependence on $\phi_{0}$ is observed between $\pi / 8$ and $3 \pi / 8$, in agreement with the findings of Ref. [15. This observation (partly) justifies $a$ posteriori the adiabatic approximation used in relation to the $\phi$ degree of freedom, described in Appendix D3. 


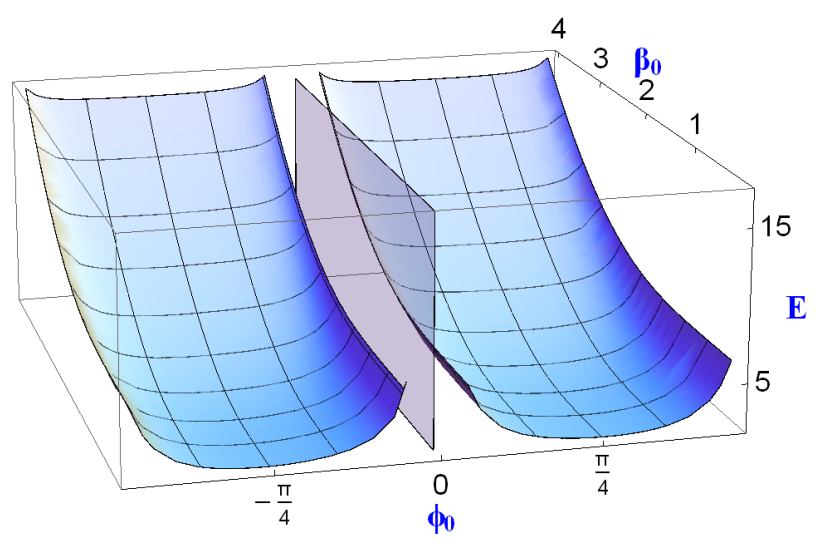

FIG. 1: (Color online) Excitation energies $E_{n_{\beta}, L}$ [Eq. 12] ] for $n_{\beta}=0$ and $L=2$ are shown as a function of the free parameters $\beta_{0}$ and $\phi_{0}$. All quantities shown are dimensionless. See subsection II.C for further discussion.

\section{D. $B(E L)$ transition rates}

The electric quadrupole and octupole operators are (Eq. (6-63) of [1])

$$
\begin{gathered}
T_{\mu}^{(E 2)}=t_{2} \beta_{2} \mathcal{D}_{0, \mu}^{(2)}(\theta)=t_{2} \sqrt{\frac{B}{B_{2}}} \tilde{\beta} \cos \phi \mathcal{D}_{0, \mu}^{(2)}(\theta), \\
T_{\mu}^{(E 3)}=t_{3} \beta_{3} \mathcal{D}_{0, \mu}^{(3)}(\theta)=t_{3} \sqrt{\frac{B}{B_{3}}} \tilde{\beta} \sin \phi \mathcal{D}_{0, \mu}^{(3)}(\theta),
\end{gathered}
$$

with

$$
t_{2}=\frac{3 Z e}{4 \pi} R^{2}, \quad t_{3}=\frac{3 Z e}{4 \pi} R^{3},
$$

where $R$ is the effective radius of the nucleus, while the electric dipole operator reads [51]

$$
T_{\mu}^{(E 1)}=t_{1} \beta_{2} \beta_{3} \mathcal{D}_{0, \mu}^{(1)}(\theta)=t_{1} \frac{B}{\sqrt{B_{2} B_{3}}} \tilde{\beta}^{2} \frac{\sin 2 \phi}{2} \mathcal{D}_{0, \mu}^{(1)}(\theta) .
$$

The total wave function reads

$$
\begin{aligned}
& \Phi_{L, M, q}^{ \pm}(\tilde{\beta}, \phi, \theta)=\left(\beta_{2} \beta_{3}\right)^{-3 / 2} N_{\tilde{\beta}} F_{n_{\beta}}^{L}(\tilde{\beta}) N_{\phi} \\
& \frac{\left(\chi_{n_{\phi}}\left(\tilde{\phi}^{+}\right) \pm \chi_{n_{\phi}}\left(\tilde{\phi}^{-}\right)\right)}{\sqrt{2}} \sqrt{\frac{2 L+1}{32 \pi^{2}}}\left(1 \pm(-1)^{L}\right) \mathcal{D}_{0, M}^{L}(\theta),
\end{aligned}
$$

where $q$ stands for the rest of the quantum numbers $\left(n_{\beta}\right.$, $\left.n_{\phi}\right)$. Since in what follows only bands with $n_{\phi}=0$ will be considered, in the remainder of the paper, as well as in the Appendices, we simplify the notation by using $n$ instead of $n_{\beta}$. As a result, in what follows, $n_{i}$ and $n_{f}$ indicate the initial and final values of $n_{\beta}$, while $q_{i}$ and $q_{f}$ denote $n_{i}$ and $n_{f}$ respectively, with $n_{\phi}=0$ at all times.
$B(E L)$ transition rates are given by

$$
B\left(E L ; L_{i} q_{i} \rightarrow L_{f} q_{f}\right)=\frac{\left|\left\langle L_{f} q_{f} \| T^{(E L)}|| L_{i} q_{i}\right\rangle\right|^{2}}{\left(2 L_{i}+1\right)},
$$

where the reduced matrix element is obtained through the Wigner-Eckart theorem

$$
\begin{aligned}
\left\langle L_{f} \mu_{f} q_{f}\left|T_{\mu}^{(E L)}\right| L_{i} \mu_{i} q_{i}\right\rangle= & \\
& \frac{\left(L_{i} L L_{f} \mid \mu_{i} \mu \mu_{f}\right)}{\sqrt{2 L_{f}+1}}\left\langle L_{f} q_{f}\left\|T^{E L}\right\| L_{i} q_{i}\right\rangle .
\end{aligned}
$$

In Eq. 21) the integration over the Euler angles $\theta$ involves standard integrals over three Wigner functions calculated in Appendix B, while the rest of the integrations are performed over $\iint \beta_{2}^{3} d \beta_{2} \beta_{3}^{3} d \beta_{3}$, where the $\beta_{2}^{3}$, $\beta_{3}^{3}$ factors come from the volume element and cancel with the first factor of Eq. (20). Using Eqs. (4) and (5), as well as the relevant Jacobian, one finds (up to constant factors) that the integration is over $\iint d \beta_{2} d \beta_{3}=$ $\frac{B}{\sqrt{B_{2} B_{3}}} \iint \tilde{\beta} d \tilde{\beta} d \phi$.

Relevant integrals over $\phi$ are calculated in Appendix A, while integrals over $\tilde{\beta}$ are determined in Appendix C. The final results for matrix elements are summarized here.

Matrix elements of $T^{(E 2)}$ between positive parity levels within the ground state band $\left(n_{i}=n_{f}=0\right)$ read

$$
\begin{gathered}
\left\langle L_{f} q_{f}\left\|T^{E 2}\right\| L_{i} q_{i}\right\rangle_{S \rightarrow S}=t_{2} \sqrt{\frac{B}{B_{2}}} e^{-\frac{1}{4 b^{2}}} \frac{\cos \phi_{0}+e^{-b^{2} \phi_{0}^{2}}}{1+e^{-b^{2} \phi_{0}^{2}}} \\
\sqrt{2 L_{i}+1}\left(L_{i} 2 L_{f} \mid 000\right) \frac{\Gamma\left(\frac{a_{i}}{2}+\frac{a_{f}}{2}+\frac{3}{2}\right)}{\sqrt{\Gamma\left(a_{i}+1\right) \Gamma\left(a_{f}+1\right)}} \cdot
\end{gathered}
$$

Matrix elements of $T^{(E 2)}$ from a positive parity level of the first excited band $\left(n_{i}=1\right)$ to a positive parity level of the ground state band $\left(n_{f}=0\right)$ are

$$
\begin{array}{r}
\left\langle L_{f} q_{f}\left\|T^{E 2}\right\| L_{i} q_{i}\right\rangle_{S \rightarrow S}=t_{2} \sqrt{\frac{B}{B_{2}}} \\
e^{-\frac{1}{4 b^{2}}} \frac{\cos \phi_{0}+e^{-b^{2} \phi_{0}^{2}}}{1+e^{-b^{2} \phi_{0}^{2}}} \sqrt{2 L_{i}+1}\left(L_{i} 2 L_{f} \mid 000\right) \\
\frac{\Gamma\left(\frac{a_{i}}{2}+\frac{a_{f}}{2}+\frac{3}{2}\right) \Gamma\left(\frac{a_{i}}{2}-\frac{a_{f}}{2}+\frac{1}{2}\right)}{\sqrt{\Gamma\left(a_{i}+2\right) \Gamma\left(a_{f}+1\right)} \Gamma\left(\frac{a_{i}}{2}-\frac{a_{f}}{2}-\frac{1}{2}\right)} .
\end{array}
$$

Matrix elements of $T^{(E 2)}$ between negative parity levels within the lowest band $\left(n_{i}=n_{f}=0\right)$ have the form

$$
\begin{gathered}
\left\langle L_{f} q_{f}\left\|T^{E 2}\right\| L_{i} q_{i}\right\rangle_{A \rightarrow A}=t_{2} \sqrt{\frac{B}{B_{2}}} e^{-\frac{1}{4 b^{2}}} \frac{\cos \phi_{0}-e^{-b^{2} \phi_{0}^{2}}}{1-e^{-b^{2} \phi_{0}^{2}}} \\
\sqrt{2 L_{i}+1}\left(L_{i} 2 L_{f} \mid 000\right) \frac{\Gamma\left(\frac{a_{i}}{2}+\frac{a_{f}}{2}+\frac{3}{2}\right)}{\sqrt{\Gamma\left(a_{i}+1\right) \Gamma\left(a_{f}+1\right)}} \cdot
\end{gathered}
$$

Matrix elements of $T^{(E 3)}$ between a positive parity level of the ground state band and a negative parity level 
of the lowest band, or vice versa, read

$$
\begin{gathered}
\left\langle L_{f} q_{f}\left\|T^{E 3}\right\| L_{i} q_{i}\right\rangle=t_{3} \sqrt{\frac{B}{B_{3}}} \frac{e^{-\frac{1}{4 b^{2}}} \sin \phi_{0}}{\sqrt{1-e^{-2 b^{2} \phi_{0}^{2}}}} \sqrt{2 L_{i}+1} \\
\left(L_{i} 3 L_{f} \mid 000\right) \frac{\Gamma\left(\frac{a_{i}}{2}+\frac{a_{f}}{2}+\frac{3}{2}\right)}{\sqrt{\Gamma\left(a_{i}+1\right) \Gamma\left(a_{f}+1\right)}} .
\end{gathered}
$$

Matrix elements of $T^{(E 1)}$ between a positive parity level of the ground state band and a negative parity level of the lowest band, or vice versa, are

$$
\begin{gathered}
\left\langle L_{f} q_{f} \| T^{E 1}|| L_{i} q_{i}\right\rangle_{S \rightarrow S}=\frac{1}{2} t_{1} \frac{B}{\sqrt{B_{2} B_{3}}} \frac{e^{-\frac{1}{b^{2}}} \sin 2 \phi_{0}}{\sqrt{1-e^{-2 b^{2} \phi_{0}^{2}}}} \\
\sqrt{2 L_{i}+1}\left(L_{i} 1 L_{f} \mid 000\right) \frac{\Gamma\left(\frac{a_{i}}{2}+\frac{a_{f}}{2}+2\right)}{\sqrt{\Gamma\left(a_{i}+1\right) \Gamma\left(a_{f}+1\right)}} .
\end{gathered}
$$

The final results for $B(E L)$ s are summarized here.

$B(E 2)$ s between positive parity levels within the ground state band $\left(n_{i}=n_{f}=0\right)$ read

$$
\begin{aligned}
B\left(E 2 ; L_{i} q_{i} \rightarrow\right. & \left.L_{f} q_{f}\right)_{S \rightarrow S}=t_{2}^{2} \frac{B}{B_{2}} \\
& e^{-\frac{1}{2 b^{2}}} \frac{\left(\cos \phi_{0}+e^{-b^{2} \phi_{0}^{2}}\right)^{2}}{\left(1+e^{\left.-b^{2} \phi_{0}^{2}\right)^{2}}\right.} \\
& \left(L_{i} 2 L_{f} \mid 000\right)^{2} \frac{\left[\Gamma\left(\frac{a_{i}}{2}+\frac{a_{f}}{2}+\frac{3}{2}\right)\right]^{2}}{\Gamma\left(a_{i}+1\right) \Gamma\left(a_{f}+1\right)} .
\end{aligned}
$$

$B(E 2) \mathrm{s}$ from a positive parity level of the first excited band $\left(n_{i}=1\right)$ to a positive parity level of the ground state band $\left(n_{f}=0\right)$ are

$$
\begin{aligned}
& B\left(E 2 ; L_{i} q_{i} \rightarrow L_{f} q_{f}\right)_{S \rightarrow S}=t_{2}^{2} \frac{B}{B_{2}} \\
& e^{-\frac{1}{2 b^{2}}} \frac{\left(\cos \phi_{0}+e^{-b^{2} \phi_{0}^{2}}\right)^{2}}{\left(1+e^{-b^{2} \phi_{0}^{2}}\right)^{2}}\left(L_{i} 2 L_{f} \mid 000\right)^{2} \\
& \frac{\left[\Gamma\left(\frac{a_{i}}{2}+\frac{a_{f}}{2}+\frac{3}{2}\right) \Gamma\left(\frac{a_{i}}{2}-\frac{a_{f}}{2}+\frac{1}{2}\right)\right]^{2}}{\Gamma\left(a_{i}+2\right) \Gamma\left(a_{f}+1\right)\left[\Gamma\left(\frac{a_{i}}{2}-\frac{a_{f}}{2}-\frac{1}{2}\right)\right]^{2}} .
\end{aligned}
$$

$B(E 2)$ s between negative parity levels within the lowest band $\left(n_{i}=n_{f}=0\right)$ have the form

$$
\begin{aligned}
B\left(E 2 ; L_{i} q_{i} \rightarrow\right. & \left.L_{f} q_{f}\right)_{A \rightarrow A}=t_{2}^{2} \frac{B}{B_{2}} \\
& e^{-\frac{1}{2 b^{2}}} \frac{\left(\cos \phi_{0}-e^{-b^{2} \phi_{0}^{2}}\right)^{2}}{\left(1-e^{-b^{2} \phi_{0}^{2}}\right)^{2}} \\
& \left(L_{i} 2 L_{f} \mid 000\right)^{2} \frac{\left[\Gamma\left(\frac{a_{i}}{2}+\frac{a_{f}}{2}+\frac{3}{2}\right)\right]^{2}}{\Gamma\left(a_{i}+1\right) \Gamma\left(a_{f}+1\right)} .
\end{aligned}
$$

$B(E 3)$ s between a positive parity level of the ground state band and a negative parity level of the lowest band, or vice versa, read

$$
\begin{aligned}
B\left(E 3 ; L_{i} q_{i} \rightarrow\right. & \left.L_{f} q_{f}\right)=t_{3}^{2} \frac{B}{B_{3}} \frac{e^{-\frac{1}{2 b^{2}}} \sin ^{2} \phi_{0}}{\left(1-e^{-2 b^{2} \phi_{0}^{2}}\right)} \\
\left(L_{i} 3 L_{f} \mid 000\right)^{2} & \frac{\left[\Gamma\left(\frac{a_{i}}{2}+\frac{a_{f}}{2}+\frac{3}{2}\right)\right]^{2}}{\Gamma\left(a_{i}+1\right) \Gamma\left(a_{f}+1\right)} .
\end{aligned}
$$

$B(E 1)$ s between a positive parity level of the ground state band and a negative parity level of the lowest band, or vice versa, are

$$
\begin{array}{r}
B\left(E 1 ; L_{i} q_{i} \rightarrow L_{f} q_{f}\right)=\frac{1}{4} t_{1}^{2} \frac{B^{2}}{B_{2} B_{3}} \frac{e^{-\frac{2}{b^{2}}} \sin ^{2} 2 \phi_{0}}{\left(1-e^{\left.-2 b^{2} \phi_{0}^{2}\right)}\right.} \\
\left(L_{i} 1 L_{f} \mid 000\right)^{2} \frac{\left[\Gamma\left(\frac{a_{i}}{2}+\frac{a_{f}}{2}+2\right)\right]^{2}}{\Gamma\left(a_{i}+1\right) \Gamma\left(a_{f}+1\right)} .
\end{array}
$$

In Ref. [46] it has been pointed out that $B(E 1)$ s for transitions from positive parity levels $L$ of the ground state band to negative parity levels $L-1$ of the lowest band and $B(E 1)$ s for transitions in the opposite direction, i.e. from negative parity levels $L-1$ of the lowest band to positive parity levels $L$ of the ground state band, should be of the same order, as seen experimentally. This condition is clearly fulfilled by Eq. 32 .

\section{NUMERICAL RESULTS}

\section{A. Spectra}

In the spectra of the AQOA model with Davidson potential (AQOA-D) only the parameters $\phi_{0}$ and $\beta_{0}$ play an essential role, as seen in Eq. 12, while the quantities $E_{0}, C_{1}$, and $C_{2}$ of Eq. (16) do not enter, if we consider only bands with $n_{\phi}=0$ and normalize all energies to that of the first excited state, $E\left(2_{1}^{+}\right)$. The parameters of rms fits, using the quality measure

$$
\sigma=\sqrt{\frac{\sum_{i=1}^{n}\left(E_{i}(\exp )-E_{i}(t h)\right)^{2}}{(n-1) E\left(2_{1}^{+}\right)^{2}}},
$$

to the spectra of the $\mathrm{Ra}$ and $\mathrm{Th}$ isotopes lying at the border between the regions of octupole deformation and octupole vibrations, as well as within the former region, are shown in Table I, while in Table II the relevant spectra are shown. For ${ }^{226} \mathrm{Ra}$ and ${ }^{226} \mathrm{Th}$, the predictions of the original one-parameter $\left(\phi_{0}\right)$ AQOA with an infinite well potential (AQOA-IW), applicable at the border between the regions of octupole deformation and octupole vibrations, are shown for comparison. The following comments apply.

a) Good agreement between the theoretical predictions of AQOA-D and experimental data is obtained up to high angular momenta, both in the ground state band and in the negative parity band. The predictions for the $1^{-}$and 
TABLE I: Parameters $\phi_{0}$ and $\beta_{0}$ of the AQOA model with Davidson potential (AQOA-D) obtained from rms fits to experimental spectra of ${ }^{222} \mathrm{Ra}$ [7, 8, ${ }^{224} \mathrm{Ra}$ [7, 8, ${ }^{226} \mathrm{Ra}$ [7, 8, ${ }^{224} \mathrm{Th}$ [24, and ${ }^{226} \mathrm{Th}$ [25. The experimental $R_{4 / 2}=E\left(4_{1}^{+}\right) / E\left(2_{1}^{+}\right) \mathrm{ratios}$ are also shown. The angular momenta of the highest levels of the ground state, $\beta$ and negative parity bands included in the rms fit are labelled by $L_{g}, L_{\beta}$, and $L_{o}$ respectively, while $n$ indicates the total number of levels involved in the fit and $\sigma$ is the quality measure of Eq. 33. The theoretical predictions are obtained from the formulae mentioned in subsec. II.B . See subsec. III.A for further discussion. For completeness, the parameter $b$, and the parameter ratios $t_{3} / t_{2}$, and $B_{2} / B_{3}$, appearing in the fitting of electric transition rates, are shown, wherever known. All quantities shown are dimensionless, exept $t_{3} / t_{2}$, which is given in fm. See subsec. III.B for further discussion.

\begin{tabular}{rccccccccccc}
\hline nucleus & $R_{4 / 2}$ & $\phi_{0}$ & $\beta_{0}$ & $L_{g}$ & $L_{\beta}$ & $L_{o}$ & $n$ & $\sigma$ & $b$ & $t_{3} / t_{2}(\mathrm{fm})$ & $B_{2} / B_{3}$ \\
\hline${ }^{222} \mathrm{Ra}$ & 2.715 & 62.7 & 0.00 & 20 & 0 & 19 & 20 & 0.917 & & 7.266 & \\
${ }^{224} \mathrm{Ra}$ & 2.970 & 41.9 & 1.80 & 28 & 0 & 27 & 28 & 1.351 & 1.836 & 7.288 & 0.65 \\
${ }^{226} \mathrm{Ra}$ & 3.127 & 24.7 & 2.14 & 28 & 0 & 27 & 28 & 1.360 & & 7.309 & \\
${ }^{224} \mathrm{Th}$ & 2.896 & 67.9 & 0.79 & 18 & & 17 & 17 & 0.843 & & 7.288 & \\
${ }^{226} \mathrm{Th}$ & 3.136 & 9.5 & 0.94 & 20 & 0 & 19 & 20 & 0.994 & & 7.309 & \\
\hline
\end{tabular}

TABLE II: Comparison of theoretical predictions of the AQOA model with Davidson potential (AQOA-D, columns labelled as D) and with an infinite well potential (AQOA-IW, columns labelled as IW) to experimental data [normalized to $E\left(2_{1}^{+}\right)$] of ${ }^{222} \mathrm{Ra}$ [7, 8, ${ }^{224} \mathrm{Ra}$ [7, 8, ${ }^{226} \mathrm{Ra}$ [7, 8, ${ }^{224} \mathrm{Th}$ [24], and ${ }^{226} \mathrm{Th}$ [25]. The AQOA-D parameters are shown in Table I, while the AQOA-IW predictions have been taken from Ref. [15. The theoretical predictions for AQOA-D are obtained from the formulae mentioned in subsec. II.B . See subsec. III.A for further discussion.

\begin{tabular}{|c|c|c|c|c|c|c|c|c|c|c|c|c|}
\hline & ${ }^{222} \mathrm{Ra}$ & ${ }^{222} \mathrm{Ra}$ & ${ }^{224} \mathrm{Ra}$ & ${ }^{224} \mathrm{Ra}$ & ${ }^{226} \mathrm{Ra}$ & ${ }^{226} \mathrm{Ra}$ & ${ }^{226} \mathrm{Ra}$ & ${ }^{224} \mathrm{Th}$ & ${ }^{224} \mathrm{Th}$ & ${ }^{226} \mathrm{Th}$ & ${ }^{226} \mathrm{Th}$ & ${ }^{226} \mathrm{Th}$ \\
\hline$L^{\pi}$ & exp. & D & exp. & D & exp. & $\mathrm{D}$ & IW & exp. & $\mathrm{D}$ & exp. & $\mathrm{D}$ & IW \\
\hline $4^{+}$ & 2.72 & 3.00 & 2.97 & 3.17 & 3.13 & 3.22 & 3.09 & 2.90 & 3.09 & 3.14 & 3.22 & 3.12 \\
\hline $6^{+}$ & 4.95 & 5.59 & 5.68 & 6.21 & 6.16 & 6.45 & 5.99 & 5.45 & 5.90 & 6.20 & 6.44 & 6.10 \\
\hline $8^{+}$ & 7.58 & 8.49 & 8.94 & 9.87 & 9.89 & 10.45 & 9.56 & 8.50 & 9.17 & 10.00 & 10.42 & 9.78 \\
\hline $10^{+}$ & 10.55 & 11.58 & 12.66 & 13.94 & 14.19 & 15.02 & 13.71 & 11.97 & 12.71 & 14.41 & 14.97 & 14.08 \\
\hline $12^{+}$ & 13.82 & 14.77 & 16.74 & 18.30 & 18.93 & 20.00 & 18.42 & 15.80 & 16.42 & 19.32 & 19.93 & 18.96 \\
\hline $14^{+}$ & 17.39 & 18.04 & 21.17 & 22.85 & 24.06 & 25.31 & 23.64 & 19.97 & 20.25 & 24.68 & 25.20 & 24.38 \\
\hline $16^{+}$ & 21.21 & 21.36 & 25.90 & 27.55 & 29.52 & 30.84 & 29.38 & 24.44 & 24.16 & 30.41 & 30.69 & 30.34 \\
\hline $18^{+}$ & 25.28 & 24.70 & 30.92 & 32.34 & 35.30 & 36.54 & 35.61 & 29.20 & 28.13 & 36.50 & 36.35 & 36.81 \\
\hline $20^{+}$ & 29.57 & 28.07 & 36.22 & 37.22 & 41.38 & 42.38 & 42.33 & & & 42.90 & 42.14 & 43.80 \\
\hline $22^{+}$ & & & 41.74 & 42.15 & 47.75 & 48.32 & 49.54 & & & & & \\
\hline $24^{+}$ & & & 47.48 & 47.13 & 54.44 & 54.34 & 57.22 & & & & & \\
\hline $26^{+}$ & & & 53.41 & 52.14 & 61.42 & 60.43 & 65.38 & & & & & \\
\hline $28^{+}$ & & & 59.54 & 57.18 & 68.70 & 66.57 & 74.01 & & & & & \\
\hline $0^{+}$ & 8.23 & 8.06 & 10.86 & 10.90 & 12.19 & 12.21 & 11.23 & & 9.91 & 11.18 & 11.31 & 12.41 \\
\hline $1^{-}$ & 2.18 & 0.35 & 2.56 & 0.34 & 3.75 & 0.34 & 0.34 & 2.56 & 0.34 & 3.19 & 0.34 & 0.34 \\
\hline $3^{-}$ & 2.85 & 1.90 & 3.44 & 1.95 & 4.75 & 1.97 & 1.93 & 3.11 & 1.93 & 4.26 & 1.97 & 1.94 \\
\hline $5^{-}$ & 4.26 & 4.24 & 5.13 & 4.60 & 6.60 & 4.72 & 4.45 & 4.74 & 4.43 & 6.24 & 4.72 & 4.51 \\
\hline $7^{-}$ & 6.33 & 7.01 & 7.59 & 7.98 & 9.26 & 8.36 & 7.70 & 7.13 & 7.49 & 9.11 & 8.35 & 7.86 \\
\hline $9^{-}$ & 8.92 & 10.02 & 10.73 & 11.86 & 12.68 & 12.67 & 11.57 & 10.17 & 10.91 & 12.79 & 12.64 & 11.86 \\
\hline $11^{-}$ & 11.97 & 13.17 & 14.46 & 16.09 & 16.74 & 17.47 & 16.00 & 13.73 & 14.55 & 17.15 & 17.41 & 16.45 \\
\hline $13^{-}$ & 15.38 & 16.40 & 18.68 & 20.56 & 21.39 & 22.63 & 20.96 & 17.72 & 18.32 & 22.11 & 22.53 & 21.61 \\
\hline $15^{-}$ & 19.11 & 19.69 & 23.31 & 25.18 & 26.54 & 28.05 & 26.45 & 22.07 & 22.20 & 27.55 & 27.92 & 27.30 \\
\hline $17^{-}$ & 23.11 & 23.03 & 28.27 & 29.93 & 32.13 & 33.67 & 32.43 & 26.71 & 26.14 & 33.42 & 33.50 & 33.51 \\
\hline $19^{-}$ & 27.35 & 26.39 & 33.51 & 34.77 & 38.10 & 39.44 & 38.91 & & & 39.63 & 39.23 & 40.24 \\
\hline $21^{-}$ & & & 38.99 & 39.68 & 44.41 & 45.34 & 45.87 & & & & & \\
\hline $23^{-}$ & & & 44.67 & 44.63 & 51.03 & 51.32 & 53.32 & & & & & \\
\hline $25^{-}$ & & & 50.55 & 49.63 & 57.93 & 57.38 & 61.24 & & & & & \\
\hline $27^{-}$ & & & 56.60 & 54.66 & 65.08 & 63.49 & 69.64 & & & & & \\
\hline
\end{tabular}

$3^{-}$states are poor, since no finite barrier is used in the phi potential [55 57 .

b) When moving from the border region towards the interior of the region of octupole deformation, the parameter $\phi_{0}$ increases, in agreement with an increasing role of the octupole deformation (which is proportional to $\sin \phi_{0}$ ), while the parameter $\beta_{0}$ decreases. In parallel, a decreasing role of the quadrupole deformation is revealed by the decreasing $R_{4 / 2}$ ratios.

c) The agreement between the predictions of AQOAIW and the data is comparable to that of AQOA-D, except at high angular momenta, where AQOA-D is closer 
TABLE III: Matrix elements of electric transitions in ${ }^{224} \mathrm{Ra}$. The experimental data, in units of $e \mathrm{fm}, e \mathrm{fm}^{2}, e \mathrm{fm}^{3}$ for $\mathrm{E} 1$, E2, E3 respectively, have been taken from Ref. [21, while the theoretical predictions have been obtained using the formulae of subsec. II.D in the way described in subsec. III.B, where further discussion is given.

\begin{tabular}{lll}
\hline m.e. & exp. & th. \\
\hline$\left\langle 0^{+}\|E 2\| 2^{+}\right\rangle$ & $199 \pm 3$ & 196 \\
$\left\langle 2^{+}\|E 2\| 4^{+}\right\rangle$ & $315 \pm 6$ & 323 \\
$\left\langle 4^{+}\|E 2\| 6^{+}\right\rangle$ & $405 \pm 15$ & 426 \\
$\left\langle 6^{+}\|E 2\| 8^{+}\right\rangle$ & $500 \pm 60$ & 525 \\
$\left\langle 1^{-}\|E 2\| 3^{-}\right\rangle$ & $230 \pm 11$ & 236 \\
$\left\langle 3^{-}\|E 2\| 5^{-}\right\rangle$ & $410 \pm 60$ & 334 \\
$\left\langle 0^{+}\|E 2\| 2_{\gamma}^{+}\right\rangle$ & $23 \pm 4$ & 36 \\
$\left\langle 0^{+}\|E 3\| 3^{-}\right\rangle$ & $940 \pm 30$ & 1006 \\
$\left\langle 2^{+}\|E 3\| 1^{-}\right\rangle$ & $1370 \pm 140$ & 1137 \\
$\left\langle 2^{+}\|E 3\| 3^{-}\right\rangle$ & $<4000$ & 1176 \\
$\left\langle 2^{+}\|E 3\| 5^{-}\right\rangle$ & $1410 \pm 190$ & 1594 \\
$\left\langle 0^{+}\|E 1\| 1^{-}\right\rangle$ & $<0.018$ & 0.013 \\
$\left\langle 2^{+}\|E 1\| 1^{-}\right\rangle$ & $<0.03$ & 0.018 \\
$\left\langle 2^{+}\|E 1\| 3^{-}\right\rangle$ & $0.026 \pm 0.005$ & 0.023 \\
$\left\langle 4^{+}\|E 1\| 5^{-}\right\rangle$ & $0.030 \pm 0.010$ & 0.032 \\
$\left\langle 6^{+}\|E 1\| 7^{-}\right\rangle$ & $<0.10$ & 0.042 \\
\hline
\end{tabular}

to the data, due to the term $\beta_{0}^{4}$ appearing in Eq. 12 , which moderates the increase of the energy with $L$.

\section{B. Transitions}

With the parameters $\phi_{0}$ and $\beta_{0}$ determined from the spectra, we now turn attention to electromagnetic transition rates, following the procedure described below.

a) From Eqs. 28 and 29 it is clear that ratios of $B(E 2)_{S \rightarrow S}$ s involve only the parameters $\phi_{0}$ and $\beta_{0}$, thus they are already fixed. The same holds separately for ratios of $B(E 2)_{A \rightarrow A} \mathrm{~s}$, or $B(E 3) \mathrm{s}$, or $B(E 1) \mathrm{s}$, as seen from Eqs. 30 , 31, and (32) respectively.

b) Ratios of $B(E 2)_{S \rightarrow S}$ S over $B(E 2)_{A \rightarrow A}$ s involve in addition the parameter $b$, which can then be determined from such ratios, as seen from Eqs. 28) and (30).

c) Ratios of $B(E 3)$ s over $B(E 2)$ s involve in addition the ratio $t_{3} / t_{2}$, which can be determined from the nuclear radius (see subsec. III.B.2), and the ratio $B_{2} / B_{3}$, which can be determined from the $B(E 3) / B(E 2)$ ratios, as seen from Eqs. (31) and 28.

This procedure can be tested against the recently measured transition matrix elements of ${ }^{224} \mathrm{Ra}$ [21], shown in Table III.

\section{E2 transitions}

The ratio of any $E 2_{A \rightarrow A}$ matrix element [Eq. 25] over any $E 2_{S \rightarrow S}$ matrix element [Eq. [23] contains the ratio

$$
r=\frac{\left(\cos \phi_{0}-a\right)(1+a)}{\left(\cos \phi_{0}+a\right)(1-a)}, \quad a=e^{-b^{2} \phi_{0}^{2}} .
$$

In the case of ${ }^{224} \mathrm{Ra}$, the ratios of experimental matrix elements $\frac{3^{-} \rightarrow 1^{-}}{2^{+} \rightarrow 0^{+}}, \frac{3^{-} \rightarrow 1^{-}}{4^{+} \rightarrow 2^{+}}, \frac{3^{-} \rightarrow 1^{-}}{6^{+} \rightarrow 4^{+}}, \frac{3^{-} \rightarrow 1^{-}}{8^{+} \rightarrow 6^{+}}$lead to $r=$ $0.852,0.887,0.909,0.908$, i.e., to an average value of 0.889 .

Solving for $a$ one obtains the quadratic equation

$$
(r-1) a^{2}+(r+1)\left(\cos \phi_{0}-1\right) a+(1-r) \cos \phi_{0}=0,
$$

having the solution

$$
\begin{aligned}
a= & \frac{1}{2(r-1)}\left[-(r+1)\left(\cos \phi_{0}-1\right)\right. \\
& \left. \pm \sqrt{(r+1)^{2}\left(\cos \phi_{0}-1\right)^{2}+4(r-1)^{2} \cos \phi_{0}}\right] .
\end{aligned}
$$

Since from Eq. (34) one has

$$
b^{2}=-\frac{\ln a}{\phi_{0}^{2}},
$$

$\ln a$ has to be negative for $b$ to be real. Since $r<1$, we see that in Eq. (36) only the negative sign is allowed in order to have $a>0$, leading in the case of ${ }^{224} \mathrm{Ra}$ to $a=0.1647$ and $b=1.836$.

One can then keep

$$
F=t_{2} \sqrt{B / B_{2}}
$$

as an overall constant for all E2 transition matrix elements, and determine it through rms fitting to the experimental data of the transitions with $n_{i}=n_{f}=0$, obtaining $F=127.20$ and the $E 2$ predictions reported in Table III. Then from Eq. 24) one can calculate also the transitions with $n_{i}=1, n_{f}=0$, one of which is also reported in Table III.

\section{E3 transitions}

For the coefficients $t_{2}$ and $t_{3}$ one can use Eq. (18), leading to

$$
\frac{t_{3}}{t_{2}}=R
$$

where $R$ is the nuclear radius, given by 58

$$
R=r_{0} A^{1 / 3}, \quad r_{0}=1.2 \mathrm{fm}
$$

with $A$ being the mass number of the nucleus. Thus in the case of ${ }^{224} \mathrm{Ra}$ one has $R=7.2878 \mathrm{fm}$.

One can then determine the ratio $\frac{B_{2}}{B_{3}}$ from any ratio of E3 matrix element over $E 2$ matrix element, since each of these ratios contains the quantity $\left(t_{3} / t_{2}\right) \sqrt{B_{2} / B_{3}}$, as seen from Eqs. 26, 23, 25. In the case of ${ }^{224} \mathrm{Ra}$, six E2 matrix elements and three $E 3$ matrix elements 
are known. Considering all 18 possible ratios, we get an average value of $\left(t_{3} / t_{2}\right) \sqrt{B_{2} / B_{3}}=5.876$, leading to $B_{2} / B_{3}=0.65$.

By now the E3 transition matrix elements have been completely determined. The overall constant

$$
F^{\prime}=t_{3} \sqrt{B / B_{3}}
$$

appearing in this case is connected to the overall constant $F$ through

$$
\frac{F^{\prime}}{F}=\frac{t_{3}}{t_{2}} \sqrt{\frac{B_{2}}{B_{3}}}=R \sqrt{\frac{B_{2}}{B_{3}}},
$$

leading to $F^{\prime}=747.47$ and to the $E 3$ matrix elements given in Table III.

\section{E1 transitions}

In the case of $E 1$ matrix elements, the quantity

$$
F^{\prime \prime}=\frac{1}{2} t_{1} \frac{B}{\sqrt{B_{2} B_{3}}} \frac{e^{-\frac{1}{b^{2}}} \sin 2 \phi_{0}}{\sqrt{1-e^{-2 b^{2} \phi_{0}^{2}}}}
$$

can be treated as an overall constant, determined in the case of ${ }^{224} \mathrm{Ra}$ by rms fitting to the two known transitions to be $F^{\prime \prime}=2.67610^{-3}$ and providing the predictions given in Table III.

\section{CONCLUSIONS}

The analytic quadrupole octupole axially symmetric model with an infinite well potential (AQOA-IW) had successfully predicted the border between the regions of octupole deformation and octupole vibrations in the light actinides, identifying ${ }^{226} \mathrm{Ra}$ and ${ }^{226} \mathrm{Th}$ as border nuclei [15, with heavier isotopes corresponding to octupole vibrations and lighter isotopes exhibiting octupole deformation. The AQOA-IW model involved only one free parameter, $\phi_{0}$, expressing the relative presence of quadrupole vs. octupole deformation, while a parameterfree version has also been developed later 59 .

In the present work, the infinite well potential is substituted by a Davidson potential, resulting in the AQOA$\mathrm{D}$ model, which is able to deviate from the border line into the region of octupole deformation. This is achieved through the extra parameter $\beta_{0}$, the position of the minimum of the Davidson potential, which is increasing with increasing $R_{4 / 2}$ ratios, as it is known from its use in the description of quadrupole deformed nuclei 23 .

Within the AQOA-D model, analytic expressions for energy spectra and $\mathrm{B}(\mathrm{E} 1), \mathrm{B}(\mathrm{E} 2), \mathrm{B}(\mathrm{E} 3)$ transition rates are derived. Then the following path is taken.

a) The spectra of ${ }^{222-226} \mathrm{Ra}$ and ${ }^{224,226} \mathrm{Th}$ [normalized to $E\left(2_{1}^{+}\right)$] are well reproduced in terms of the above mentioned two parameters $\phi_{0}$ and $\beta_{0}$. b) The parameter $b$, related to the harmonic oscillator potential used in the $\phi$ degree of freedom, can be determined from the ratio of any $E 2$ matrix element between negative parity states over any $E 2$ matrix element between positive parity states, fixing the determination of all $E 2$ transitions up to an overall scale factor.

c) The ratio of mass parameters $B_{2} / B_{3}$ can be determined from the ratio of any $E 3$ matrix element over any E2 matrix element, while the ratio of transition coefficients $t_{2} / t_{3}$ is fixed by the nuclear radius. As a result, the determination of all $E 2$ and $E 3$ transitions is fixed, without any additional overall scale factor.

d) E1 transitions are also fixed, up to another scale factor.

The recently measured $B(E L)$ transition rates of ${ }^{224} \mathrm{Ra}$ 21, presenting stable octupole deformation, provide a successful test for the model. It is clear that for other nuclei, the minimum set of data needed includes

a) A few energy levels of both positive and negative parity, from which the parameters $\phi_{0}$ and $\beta_{0}$ can be determined.

b) At least one $E 2$ transition between positive parity states and one $E 2$ transition between negative parity states, from which the parameter $b$ can be determined.

c) At least one E3 transition, from which, in combination with the $E 2$ transitions of b), the parameter ratio $B_{2} / B_{3}$ can be determined.

From these pieces of data

a) The spectrum (leaving out the $\gamma$ bands) is determined up to an overall scale factor.

b) All relevant $E 2$ and $E 3$ transitions are determined up to an overall scale factor.

c) All relevant $E 1$ transitions are determined up to another overall scale factor.

It is of interest to apply the present model in the actinides close to ${ }^{240} \mathrm{Pu}$, in which a second order shape phase transition from octupole-nondeformed to octupoledeformed shapes has been recently found [60, while octupole bands have been described 61 using supersymmetric quantum mechanics. The light rare earths, in which octupole bands have been considered recently both by the Bizzeti and Bizzeti-Sona approach 62 and within density functional theory 63, are also of special interest. A successful application of the AQOA-IW model to ${ }^{148} \mathrm{Nd}$ has already been given in Ref. [64.

\section{Acknowledgements}

Financial support from the Bulgarian National Science Fund under contract No. DFNI-E02/6 and by the Scientific Research Projects Coordination Unit of Istanbul University under Project No 50822 is gratefully acknowledged. 


\section{Appendix A. $\phi$ integrals}

Since we confine ourselves to states with $n_{\phi}=0$, this quantum number is omitted in the notation of the wave functions, which then carry only the subscript $i(f)$ for the initial (final) state.

For symmetric states one has

$$
X_{S}(\phi)=N_{S} \frac{\chi\left(\tilde{\phi}^{+}\right)+\chi\left(\tilde{\phi}^{-}\right)}{\sqrt{2}},
$$

while for antisymmetric states one has

$$
X_{A}(\phi)=N_{A} \frac{\chi\left(\tilde{\phi}^{+}\right)-\chi\left(\tilde{\phi}^{-}\right)}{\sqrt{2}}
$$

where $N_{S}$ and $N_{A}$ are normalization factors and, according to Eq. 15,

$$
\begin{aligned}
\chi\left(\tilde{\phi}^{+}\right)=\sqrt{\frac{b}{\sqrt{\pi}}} e^{-\frac{b^{2}}{2}\left(\phi-\phi_{0}\right)^{2}}, & \\
\chi\left(\tilde{\phi}^{-}\right) & =\sqrt{\frac{b}{\sqrt{\pi}}} e^{-\frac{b^{2}}{2}\left(\phi+\phi_{0}\right)^{2}} .
\end{aligned}
$$

\section{A0. Normalization}

For symmetric states one has

$$
\begin{aligned}
& \frac{1}{N_{S}^{2}}= \int_{-\infty}^{\infty} X_{S} X_{S} d \phi \\
&= \frac{1}{2} \int_{-\infty}^{\infty}\left(\chi\left(\phi^{+}\right)\right)^{2} d \phi+\frac{1}{2} \int_{-\infty}^{\infty}\left(\chi\left(\phi^{-}\right)\right)^{2} d \phi \\
& \quad+\int_{-\infty}^{\infty} \chi\left(\phi^{+}\right) \chi\left(\phi^{-}\right) d \phi \\
&= \frac{1}{2} \frac{b}{\sqrt{\pi}} e^{-b^{2} \phi_{0}^{2}} \int_{-\infty}^{\infty} e^{-b^{2} \phi^{2}+2 b^{2} \phi_{0} \phi} d \phi \\
&+\frac{1}{2} \frac{b}{\sqrt{\pi}} e^{-b^{2} \phi_{0}^{2}} \int_{-\infty}^{\infty} e^{-b^{2} \phi^{2}-2 b^{2} \phi_{0} \phi} d \phi \\
& \quad+\frac{b}{\sqrt{\pi}} e^{-b^{2} \phi_{0}^{2}} \int_{-\infty}^{\infty} e^{-b^{2} \phi^{2}} d \phi
\end{aligned}
$$

Using Eq. 84 of Appendix A4 we see that the integrals appearing here are of the form

$$
\int_{-\infty}^{\infty} e^{-b^{2} \phi^{2} \pm 2 b^{2} \phi_{0} \phi} d \phi=\frac{\sqrt{\pi}}{b} e^{b^{2} \phi_{0}^{2}}
$$

leading to

$$
\frac{1}{N_{S}^{2}}=1+e^{-b^{2} \phi_{0}^{2}}
$$

For antisymmetric states one has

$$
\begin{aligned}
& \frac{1}{N_{A}^{2}}= \int_{-\infty}^{\infty} X_{A} X_{A} d \phi \\
&= \frac{1}{2} \int_{-\infty}^{\infty}\left(\chi\left(\phi^{+}\right)\right)^{2} d \phi+\frac{1}{2} \int_{-\infty}^{\infty}\left(\chi\left(\phi^{-}\right)\right)^{2} d \phi \\
& \quad-\int_{-\infty}^{\infty} \chi\left(\phi^{+}\right) \chi\left(\phi^{-}\right) d \phi \\
&=\frac{1}{2} \frac{b}{\sqrt{\pi}} e^{-b^{2} \phi_{0}^{2}} \int_{-\infty}^{\infty} e^{-b^{2} \phi^{2}+2 b^{2} \phi_{0} \phi} d \phi \\
&+\frac{1}{2} \frac{b}{\sqrt{\pi}} e^{-b^{2} \phi_{0}^{2}} \int_{-\infty}^{\infty} e^{-b^{2} \phi^{2}-2 b^{2} \phi_{0} \phi} d \phi \\
& \quad-\frac{b}{\sqrt{\pi}} e^{-b^{2} \phi_{0}^{2}} \int_{-\infty}^{\infty} e^{-b^{2} \phi^{2}} d \phi
\end{aligned}
$$

leading in the same way as above to

$$
\frac{1}{N_{A}^{2}}=1-e^{-b^{2} \phi_{0}^{2}}
$$

\section{A1. $B(E 2) \mathbf{s}$}

The transition operator for $B(E 2) \mathrm{s}$ contains $\tilde{\beta}_{2}=$ $\tilde{\beta} \cos \phi$.

For $B(E 2)$ s between symmetric states one has

$$
\begin{gathered}
I_{\phi, S \rightarrow S}^{(E 2)}=\int_{-\infty}^{\infty} X_{S} \cos \phi X_{S} d \phi \\
=\frac{N_{S}^{2}}{2} \int_{-\infty}^{\infty}\left(\chi\left(\phi^{+}\right)\right)^{2} \cos \phi d \phi \\
+\frac{N_{S}^{2}}{2} \int_{-\infty}^{\infty}\left(\chi\left(\phi^{-}\right)\right)^{2} \cos \phi d \phi \\
+N_{S}^{2} \int_{-\infty}^{\infty} \chi\left(\phi^{+}\right) \chi\left(\phi^{-}\right) \cos \phi d \phi \\
=\frac{N_{S}^{2}}{2} \frac{b}{\sqrt{\pi}} e^{-b^{2} \phi_{0}^{2}} \int_{-\infty}^{\infty} e^{-b^{2} \phi^{2}+2 b^{2} \phi_{0} \phi} \cos \phi d \phi \\
+\frac{N_{S}^{2}}{2} \frac{b}{\sqrt{\pi}} e^{-b^{2} \phi_{0}^{2}} \int_{-\infty}^{\infty} e^{-b^{2} \phi^{2}-2 b^{2} \phi_{0} \phi} \cos \phi d \phi \\
+N_{S}^{2} \frac{b}{\sqrt{\pi}} e^{-b^{2} \phi_{0}^{2}} \int_{-\infty}^{\infty} e^{-b^{2} \phi^{2}} \cos \phi d \phi .
\end{gathered}
$$

Using Eq. 81 of Appendix A4 we see that the integrals appearing here are of the form

$$
\int_{-\infty}^{\infty} e^{-b^{2} \phi^{2} \pm 2 b^{2} \phi_{0} \phi} \cos \phi d \phi=\frac{\sqrt{\pi}}{b} e^{b^{2} \phi_{0}^{2}-\frac{1}{4 b^{2}}} \cos \phi_{0},
$$

leading to

$$
I_{\phi, S \rightarrow S}^{(E 2)}=e^{-\frac{1}{4 b^{2}}} \frac{\cos \phi_{0}+e^{-b^{2} \phi_{0}^{2}}}{1+e^{-b^{2} \phi_{0}^{2}}} .
$$


For $B(E 2) \mathrm{s}$ between antisymmetric states one has

$$
\begin{gathered}
I_{\phi, A \rightarrow A}^{(E 2)}=\int_{-\infty}^{\infty} X_{A} \cos \phi X_{A} d \phi \\
=\frac{N_{A}^{2}}{2} \int_{-\infty}^{\infty}\left(\chi\left(\phi^{+}\right)\right)^{2} \cos \phi d \phi \\
\quad+\frac{N_{A}^{2}}{2} \int_{-\infty}^{\infty}\left(\chi\left(\phi^{-}\right)\right)^{2} \cos \phi d \phi \\
\quad-N_{A}^{2} \int_{-\infty}^{\infty} \chi\left(\phi^{+}\right) \chi\left(\phi^{-}\right) \cos \phi d \phi \\
=\frac{N_{A}^{2}}{2} \frac{b}{\sqrt{\pi}} e^{-b^{2} \phi_{0}^{2}} \int_{-\infty}^{\infty} e^{-b^{2} \phi^{2}+2 b^{2} \phi_{0} \phi} \cos \phi d \phi \\
+\frac{N_{A}^{2}}{2} \frac{b}{\sqrt{\pi}} e^{-b^{2} \phi_{0}^{2}} \int_{-\infty}^{\infty} e^{-b^{2} \phi^{2}-2 b^{2} \phi_{0} \phi} \cos \phi d \phi \\
-N_{A}^{2} \frac{b}{\sqrt{\pi}} e^{-b^{2} \phi_{0}^{2}} \int_{-\infty}^{\infty} e^{-b^{2} \phi^{2}} \cos \phi d \phi
\end{gathered}
$$

leading in the same way as above to

$$
I_{\phi, A \rightarrow A}^{(E 2)}=e^{-\frac{1}{4 b^{2}}} \frac{\cos \phi_{0}-e^{-b^{2} \phi_{0}^{2}}}{1-e^{-b^{2} \phi_{0}^{2}}}
$$

For $B(E 2)$ s between symmetric and antisymmetric states one has

$$
\begin{gathered}
I_{\phi, S \rightarrow A}^{(E 2)}=\int_{-\infty}^{\infty} X_{S} \cos \phi X_{A} d \phi \\
=\frac{N_{S} N_{A}}{2} \int_{-\infty}^{\infty}\left(\chi\left(\phi^{+}\right)\right)^{2} \cos \phi d \phi \\
\quad-\frac{N_{S} N_{A}}{2} \int_{-\infty}^{\infty}\left(\chi\left(\phi^{-}\right)\right)^{2} \cos \phi d \phi \\
=\frac{N_{S} N_{A}}{2} \frac{b}{\sqrt{\pi}} e^{-b^{2} \phi_{0}^{2}} \int_{-\infty}^{\infty} e^{-b^{2} \phi^{2}+2 b^{2} \phi_{0} \phi} \cos \phi d \phi \\
-\frac{N_{S} N_{A}}{2} \frac{b}{\sqrt{\pi}} e^{-b^{2} \phi_{0}^{2}} \int_{-\infty}^{\infty} e^{-b^{2} \phi^{2}-2 b^{2} \phi_{0} \phi} \cos \phi d \phi,
\end{gathered}
$$

leading to

$$
I_{\phi, S \rightarrow A}^{(E 2)}=0 .
$$

In the same way one also finds

$$
I_{\phi, A \rightarrow S}^{(E 2)}=0 .
$$

\section{A2. $B(E 3) \mathbf{s}$}

The transition operator for $B(E 3) \mathrm{s}$ contains $\tilde{\beta}_{3}=$ $\tilde{\beta} \sin \phi$.
For $B(E 3) \mathrm{s}$ between symmetric states one has

$$
\begin{gathered}
I_{\phi, S \rightarrow S}^{(E 3)}=\int_{-\infty}^{\infty} X_{S} \sin \phi X_{S} d \phi \\
=\frac{N_{S}^{2}}{2} \int_{-\infty}^{\infty}\left(\chi\left(\phi^{+}\right)\right)^{2} \sin \phi d \phi \\
+\frac{N_{S}^{2}}{2} \int_{-\infty}^{\infty}\left(\chi\left(\phi^{-}\right)\right)^{2} \sin \phi d \phi \\
+N_{S}^{2} \int_{-\infty}^{\infty} \chi\left(\phi^{+}\right) \chi\left(\phi^{-}\right) \sin \phi d \phi \\
=\frac{N_{S}^{2}}{2} \frac{b}{\sqrt{\pi}} e^{-b^{2} \phi_{0}^{2}} \int_{-\infty}^{\infty} e^{-b^{2} \phi^{2}+2 b^{2} \phi_{0} \phi} \sin \phi d \phi \\
+\frac{N_{S}^{2}}{2} \frac{b}{\sqrt{\pi}} e^{-b^{2} \phi_{0}^{2}} \int_{-\infty}^{\infty} e^{-b^{2} \phi^{2}-2 b^{2} \phi_{0} \phi} \sin \phi d \phi \\
+N_{S}^{2} \frac{b}{\sqrt{\pi}} e^{-b^{2} \phi_{0}^{2}} \int_{-\infty}^{\infty} e^{-b^{2} \phi^{2}} \sin \phi d \phi .
\end{gathered}
$$

Using Eq. 83 of Appendix A4 we see that the integrals appearing here are of the form

$$
\int_{-\infty}^{\infty} e^{-b^{2} \phi^{2} \pm 2 b^{2} \phi_{0} \phi} \sin \phi d \phi= \pm \frac{\sqrt{\pi}}{b} e^{b^{2} \phi_{0}^{2}-\frac{1}{4 b^{2}}} \sin \phi_{0},
$$

leading to

$$
I_{\phi, S \rightarrow S}^{(E 3)}=0 .
$$

For $B(E 3)$ s between antisymmetric states one has

$$
\begin{gathered}
I_{\phi, A \rightarrow A}^{(E 3)}=\int_{-\infty}^{\infty} X_{A} \sin \phi X_{A} d \phi \\
=\frac{N_{S}^{2}}{2} \int_{-\infty}^{\infty}\left(\chi\left(\phi^{+}\right)\right)^{2} \sin \phi d \phi \\
\quad+\frac{N_{S}^{2}}{2} \int_{-\infty}^{\infty}\left(\chi\left(\phi^{-}\right)\right)^{2} \sin \phi d \phi \\
-N_{S}^{2} \int_{-\infty}^{\infty} \chi\left(\phi^{+}\right) \chi\left(\phi^{-}\right) \sin \phi d \phi \\
=\frac{N_{S}^{2}}{2} \frac{b}{\sqrt{\pi}} e^{-b^{2} \phi_{0}^{2}} \int_{-\infty}^{\infty} e^{-b^{2} \phi^{2}+2 b^{2} \phi_{0} \phi} \sin \phi d \phi \\
+\frac{N_{S}^{2}}{2} \frac{b}{\sqrt{\pi}} e^{-b^{2} \phi_{0}^{2}} \int_{-\infty}^{\infty} e^{-b^{2} \phi^{2}-2 b^{2} \phi_{0} \phi} \sin \phi d \phi \\
-N_{S}^{2} \frac{b}{\sqrt{\pi}} e^{-b^{2} \phi_{0}^{2}} \int_{-\infty}^{\infty} e^{-b^{2} \phi^{2}} \sin \phi d \phi
\end{gathered}
$$

leading in the same way as above to

$$
I_{\phi, A \rightarrow A}^{(E 3)}=0 .
$$

For $B(E 3) \mathrm{s}$ between symmetric and antisymmetric 
states one has

$$
\begin{gathered}
I_{\phi, S \rightarrow A}^{(E 3)}=\int_{-\infty}^{\infty} X_{S} \sin \phi X_{A} d \phi \\
=\frac{N_{S} N_{A}}{2} \int_{-\infty}^{\infty}\left(\chi\left(\phi^{+}\right)\right)^{2} \sin \phi d \phi \\
\quad-\frac{N_{S} N_{A}}{2} \int_{-\infty}^{\infty}\left(\chi\left(\phi^{-}\right)\right)^{2} \sin \phi d \phi \\
=\frac{N_{S} N_{A}}{2} \frac{b}{\sqrt{\pi}} e^{-b^{2} \phi_{0}^{2}} \int_{-\infty}^{\infty} e^{-b^{2} \phi^{2}+2 b^{2} \phi_{0} \phi} \sin \phi d \phi \\
-\frac{N_{S} N_{A}}{2} \frac{b}{\sqrt{\pi}} e^{-b^{2} \phi_{0}^{2}} \int_{-\infty}^{\infty} e^{-b^{2} \phi^{2}-2 b^{2} \phi_{0} \phi} \sin \phi d \phi
\end{gathered}
$$

leading to

$$
I_{\phi, S \rightarrow A}^{(E 3)}=\frac{e^{-\frac{1}{4 b^{2}}} \sin \phi_{0}}{\sqrt{1-e^{-2 b^{2} \phi_{0}^{2}}}}
$$

In the same way one finds

$$
I_{\phi, A \rightarrow S}^{(E 3)}=I_{\phi, S \rightarrow A}^{(E 3)}
$$

\section{A3. $B(E 1) \mathbf{s}$}

The transition operator for $B(E 1)$ s contains $\tilde{\beta}_{2} \tilde{\beta}_{3}=$ $\tilde{\beta} \cos \phi \tilde{\beta} \sin \phi=\tilde{\beta}^{2} \frac{\sin 2 \phi}{2}$.

For $B(E 1)$ s between symmetric states one has

$$
\begin{aligned}
I_{\phi, S \rightarrow S}^{(E 1)}= & \frac{1}{2} \int_{-\infty}^{\infty} X_{S} \sin 2 \phi X_{S} d \phi \\
= & \frac{N_{S}^{2}}{4} \int_{-\infty}^{\infty}\left(\chi\left(\phi^{+}\right)\right)^{2} \sin 2 \phi d \phi \\
& +\frac{N_{S}^{2}}{4} \int_{-\infty}^{\infty}\left(\chi\left(\phi^{-}\right)\right)^{2} \sin 2 \phi d \phi \\
& +\frac{N_{S}^{2}}{2} \int_{-\infty}^{\infty} \chi\left(\phi^{+}\right) \chi\left(\phi^{-}\right) \sin 2 \phi d \phi \\
=\frac{N_{S}^{2}}{4} & \frac{b}{\sqrt{\pi}} e^{-b^{2} \phi_{0}^{2}} \int_{-\infty}^{\infty} e^{-b^{2} \phi^{2}+2 b^{2} \phi_{0} \phi} \sin 2 \phi d \phi \\
+\frac{N_{S}^{2}}{4} & \frac{b}{\sqrt{\pi}} e^{-b^{2} \phi_{0}^{2}} \int_{-\infty}^{\infty} e^{-b^{2} \phi^{2}-2 b^{2} \phi_{0} \phi} \sin 2 \phi d \phi \\
& +\frac{N_{S}^{2}}{2} \frac{b}{\sqrt{\pi}} e^{-b^{2} \phi_{0}^{2}} \int_{-\infty}^{\infty} e^{-b^{2} \phi^{2}} \sin 2 \phi d \phi .
\end{aligned}
$$

Using Eq. 83 of Appendix A4 we see that the integrals appearing here are of the form

$$
\int_{-\infty}^{\infty} e^{-b^{2} \phi^{2} \pm 2 b^{2} \phi_{0} \phi} \sin 2 \phi d \phi= \pm \frac{\sqrt{\pi}}{b} e^{b^{2} \phi_{0}^{2}-\frac{1}{b^{2}}} \sin 2 \phi_{0}
$$

leading to

$$
I_{\phi, S \rightarrow S}^{(E 1)}=0
$$

For $B(E 1)$ s between antisymmetric states one has

$$
\begin{aligned}
I_{\phi, A \rightarrow A}^{(E 1)}= & \frac{1}{2} \int_{-\infty}^{\infty} X_{A} \sin 2 \phi X_{A} d \phi \\
= & \frac{N_{A}^{2}}{4} \int_{-\infty}^{\infty}\left(\chi\left(\phi^{+}\right)\right)^{2} \sin 2 \phi d \phi \\
& +\frac{N_{A}^{2}}{4} \int_{-\infty}^{\infty}\left(\chi\left(\phi^{-}\right)\right)^{2} \sin 2 \phi d \phi \\
& -\frac{N_{A}^{2}}{2} \int_{-\infty}^{\infty} \chi\left(\phi^{+}\right) \chi\left(\phi^{-}\right) \sin 2 \phi d \phi \\
=\frac{N_{A}^{2}}{4} & \frac{b}{\sqrt{\pi}} e^{-b^{2} \phi_{0}^{2}} \int_{-\infty}^{\infty} e^{-b^{2} \phi^{2}+2 b^{2} \phi_{0} \phi} \sin 2 \phi d \phi \\
+\frac{N_{A}^{2}}{4} & \frac{b}{\sqrt{\pi}} e^{-b^{2} \phi_{0}^{2}} \int_{-\infty}^{\infty} e^{-b^{2} \phi^{2}-2 b^{2} \phi_{0} \phi} \sin 2 \phi d \phi \\
& -\frac{N_{A}^{2}}{2} \frac{b}{\sqrt{\pi}} e^{-b^{2} \phi_{0}^{2}} \int_{-\infty}^{\infty} e^{-b^{2} \phi^{2}} \sin 2 \phi d \phi
\end{aligned}
$$

leading in the same way as above to

$$
I_{\phi, A \rightarrow A}^{(E 1)}=0 .
$$

For $B(E 1)$ s between symmetric and antisymmetric states one has

$$
\begin{gathered}
I_{\phi, S \rightarrow A}^{(E 1)}=\frac{1}{2} \int_{-\infty}^{\infty} X_{S} \sin 2 \phi X_{A} d \phi \\
=\frac{N_{S} N_{A}}{4} \int_{-\infty}^{\infty}\left(\chi\left(\phi^{+}\right)\right)^{2} \sin 2 \phi d \phi \\
\quad-\frac{N_{S} N_{A}}{4} \int_{-\infty}^{\infty}\left(\chi\left(\phi^{-}\right)\right)^{2} \sin 2 \phi d \phi \\
=\frac{N_{S} N_{A}}{4} \frac{b}{\sqrt{\pi}} e^{-b^{2} \phi_{0}^{2}} \int_{-\infty}^{\infty} e^{-b^{2} \phi^{2}+2 b^{2} \phi_{0} \phi} \sin 2 \phi d \phi \\
-\frac{N_{S} N_{A}}{4} \frac{b}{\sqrt{\pi}} e^{-b^{2} \phi_{0}^{2}} \int_{-\infty}^{\infty} e^{-b^{2} \phi^{2}-2 b^{2} \phi_{0} \phi} \sin 2 \phi d \phi,
\end{gathered}
$$

leading to

$$
I_{\phi, S \rightarrow A}^{(E 1)}=\frac{e^{-\frac{1}{b^{2}}} \sin 2 \phi_{0}}{2 \sqrt{1-e^{-2 b^{2} \phi_{0}^{2}}}} .
$$

In the same way one finds

$$
I_{\phi, A \rightarrow S}^{(E 1)}=I_{\phi, S \rightarrow A}^{(E 1)} .
$$

\section{A4. Useful integrals}

We know that (Eq. 3.897.2 of Ref. 65])

$$
\begin{aligned}
\int_{0}^{\infty} e^{-\beta x^{2}-\gamma x} \cos b x d x= & \\
\frac{1}{4} \sqrt{\frac{\pi}{b}} & \left\{e^{(\gamma-i b)^{2} /(4 \beta)}\left[1-\Phi\left(\frac{\gamma-i b}{2 \sqrt{\beta}}\right)\right]\right. \\
+e^{(\gamma+i b)^{2} /(4 \beta)} & {\left.\left[1-\Phi\left(\frac{\gamma+i b}{2 \sqrt{\beta}}\right)\right]\right\}, }
\end{aligned}
$$


where $\operatorname{Re} \beta>0, \quad b>0$, and $\Phi(x)$ is the error function, having the property

$$
\Phi(-x)=-\Phi(x) .
$$

Changing the variable into $y=-x$, Eq. (76) takes the form

$$
\begin{aligned}
-\int_{0}^{-\infty} e^{-\beta y^{2}+\gamma y} \cos b y d y & = \\
\frac{1}{4} \sqrt{\frac{\pi}{b}} & \left\{e^{(\gamma-i b)^{2} /(4 \beta)}\left[1-\Phi\left(\frac{\gamma-i b}{2 \sqrt{\beta}}\right)\right]\right. \\
+e^{(\gamma+i b)^{2} /(4 \beta)} & {\left.\left[1-\Phi\left(\frac{\gamma+i b}{2 \sqrt{\beta}}\right)\right]\right\} }
\end{aligned}
$$

Changing the symbol $y$ into $x$ and letting $\gamma \rightarrow-\gamma$, one then gets

$$
\begin{aligned}
& \int_{-\infty}^{0} e^{-\beta x^{2}-\gamma x} \cos b x d x= \\
& \frac{1}{4} \sqrt{\frac{\pi}{b}}\left\{e^{(-\gamma-i b)^{2} /(4 \beta)}\left[1-\Phi\left(\frac{-\gamma-i b}{2 \sqrt{\beta}}\right)\right]\right. \\
& \left.+e^{(-\gamma+i b)^{2} /(4 \beta)}\left[1-\Phi\left(\frac{-\gamma+i b}{2 \sqrt{\beta}}\right)\right]\right\} .
\end{aligned}
$$

Taking into account the property 77 , Eq. 79 takes the form

$$
\begin{aligned}
\int_{-\infty}^{0} e^{-\beta x^{2}-\gamma x} \cos b x d x= & \\
\frac{1}{4} \sqrt{\frac{\pi}{b}} & \left\{e^{(\gamma+i b)^{2} /(4 \beta)}\left[1+\Phi\left(\frac{\gamma+i b}{2 \sqrt{\beta}}\right)\right]\right. \\
& \left.+e^{(\gamma-i b)^{2} /(4 \beta)}\left[1+\Phi\left(\frac{\gamma-i b}{2 \sqrt{\beta}}\right)\right]\right\},
\end{aligned}
$$

Adding Eqs. (76) and (80, we get

$$
\begin{aligned}
\int_{-\infty}^{\infty} e^{-\beta x^{2}-\gamma x} \cos b x d x= & \frac{1}{2} \sqrt{\frac{\pi}{\beta}}\left(e^{\frac{(\gamma+i b)^{2}}{4 \beta}}+e^{\frac{(\gamma-i b)^{2}}{4 \beta}}\right) \\
& =\sqrt{\frac{\pi}{\beta}} e^{\frac{\gamma^{2}-b^{2}}{4 \beta}} \cos \frac{\gamma b}{2 \beta} .
\end{aligned}
$$

In a similar way, starting from the integral (Eq. 3.897.1 of Ref. [65])

$$
\begin{aligned}
\int_{0}^{\infty} e^{-\beta x^{2}-\gamma x} & \sin b x d x= \\
-\frac{i}{4} \sqrt{\frac{\pi}{b}} & \left\{e^{(\gamma-i b)^{2} /(4 \beta)}\left[1-\Phi\left(\frac{\gamma-i b}{2 \sqrt{\beta}}\right)\right]\right. \\
- & \left.e^{(\gamma+i b)^{2} /(4 \beta)}\left[1-\Phi\left(\frac{\gamma+i b}{2 \sqrt{\beta}}\right)\right]\right\},
\end{aligned}
$$

where $\operatorname{Re} \beta>0, \quad b>0$, we get

$$
\begin{aligned}
\int_{-\infty}^{\infty} e^{-\beta x^{2}-\gamma x} \sin b x d x & =\frac{i}{2} \sqrt{\frac{\pi}{\beta}}\left(e^{\frac{(\gamma+i b)^{2}}{4 \beta}}-e^{\frac{(\gamma-i b)^{2}}{4 \beta}}\right) \\
& =-\sqrt{\frac{\pi}{\beta}} e^{\frac{\gamma^{2}-b^{2}}{4 \beta}} \sin \frac{\gamma b}{2 \beta} .
\end{aligned}
$$

For normalization purposes the integral

$$
\int_{-\infty}^{\infty} e^{-\left(a x^{2}+b x+c\right)} d x=\sqrt{\frac{\pi}{a}} e^{\left(b^{2}-4 a c\right) /(4 a)}
$$

suffices.

\section{Appendix B. $\theta$ integrals}

Integrals over $\theta$ involve three Wigner functions and can be calculated using Eq. (4.6.2) of Ref. 66]

$$
\begin{gathered}
\int d^{3} \theta \mathcal{D}_{k_{1} m_{1}}^{\left(j_{1}\right)}(\theta) \mathcal{D}_{k_{2} m_{2}}^{\left(j_{2}\right)}(\theta) \mathcal{D}_{k_{3} m_{3}}^{\left(j_{3}\right)}(\theta) \\
=8 \pi^{2}\left(\begin{array}{lll}
j_{1} & j_{2} & j_{3} \\
k_{1} & k_{2} & k_{3}
\end{array}\right)\left(\begin{array}{ccc}
j_{1} & j_{2} & j_{3} \\
m_{1} & m_{2} & m_{3}
\end{array}\right) .
\end{gathered}
$$

Using the relation between 3 -j symbols and Clebsch Gordan coefficients (3.7.3) of 66

$$
\left(\begin{array}{ccc}
j_{1} & j_{2} & j_{3} \\
m_{1} & m_{2} & m_{3}
\end{array}\right)=\frac{(-1)^{j_{1}-j_{2}-m_{3}}}{\sqrt{2 j_{3}+1}}\left(j_{1} j_{2} j_{3} \mid m_{1} m_{2}-m_{3}\right)
$$

and the relation for conjugate Wigner functions (4.2.7) of 66

$$
\mathcal{D}_{k m}^{(j) *}(\theta)=(-1)^{k-m} \mathcal{D}_{-k-m}^{(j)}(\theta),
$$

one obtains

$$
\begin{aligned}
& \int d^{3} \theta \mathcal{D}_{k_{1} m_{1}}^{\left(j_{1}\right)}(\theta) \mathcal{D}_{k_{2} m_{2}}^{\left(j_{2}\right)}(\theta) \mathcal{D}_{-k_{3}-m_{3}}^{\left(j_{3}\right) *}(\theta)(-1)^{2 k_{3}} \\
= & \frac{8 \pi^{2}}{2 j_{3}+1}\left(j_{1} j_{2} j_{3} \mid k_{1} k_{2}-k_{3}\right)\left(j_{1} j_{2} j_{3} \mid m_{1} m_{2}-m_{3}\right),
\end{aligned}
$$

which by replacing $m_{3}\left(k_{3}\right)$ by $-m_{3}\left(-k_{3}\right)$ can be rewritten as

$$
\begin{aligned}
& \int d^{3} \theta \mathcal{D}_{k_{1} m_{1}}^{\left(j_{1}\right)}(\theta) \mathcal{D}_{k_{2} m_{2}}^{\left(j_{2}\right)}(\theta) \mathcal{D}_{k_{3} m_{3}}^{\left(j_{3}\right) *}(\theta)(-1)^{-2 k_{3}} \\
& =\frac{8 \pi^{2}}{2 j_{3}+1}\left(j_{1} j_{2} j_{3} \mid k_{1} k_{2} k_{3}\right)\left(j_{1} j_{2} j_{3} \mid m_{1} m_{2} m_{3}\right)
\end{aligned}
$$

\section{B1. $B(E 2) \mathbf{s}$}

In this case the integral reads

$$
\begin{aligned}
& I_{\theta}^{(E 2)}=\int d^{3} \theta \sqrt{\frac{2 L_{i}+1}{32 \pi^{2}}}\left[1 \pm(-1)^{L_{i}}\right] \mathcal{D}_{0, M_{i}}^{L_{i}}(\theta) \mathcal{D}_{0, \mu}^{(2)}(\theta) \\
& \sqrt{\frac{2 L_{f}+1}{32 \pi^{2}}}\left[1 \pm(-1)^{L_{f}}\right] \mathcal{D}_{0, M_{f}}^{L_{f} *}(\theta) .
\end{aligned}
$$

Using Eq. 89 this gives

$$
\begin{array}{r}
I_{\theta}^{(E 2)}=\frac{\left(1 \pm(-1)^{L_{i}}\right)\left(1 \pm(-1)^{L_{f}}\right)}{4} \sqrt{\frac{2 L_{i}+1}{2 L_{f}+1}} \\
\left(L_{i} 2 L_{f} \mid 000\right)\left(L_{i} 2 L_{f} \mid M_{i} \mu M_{f}\right) .
\end{array}
$$


From the $\phi$ integrals we know that non-vanishing results are obtained only in the $S \rightarrow S$ and $A \rightarrow A$ cases.

In the $S \rightarrow S$ case the two factors in the rhs of Eq. (91) have the positive signs in the place of the double signs, thus allowing only even values of $L_{i}$ and $L_{f}$, resulting in a factor of 4 in the numerator.

In the $A \rightarrow A$ case the two factors in the rhs of Eq. (91) have the negative signs in the place of the double signs, thus allowing only odd values of $L_{i}$ and $L_{f}$, resulting again in a factor of 4 in the numerator.

As a consequence, in all cases the final reasult reads

$$
I_{\theta}^{(E 2)}=\sqrt{\frac{2 L_{i}+1}{2 L_{f}+1}}\left(L_{i} 2 L_{f} \mid 000\right)\left(L_{i} 2 L_{f} \mid M_{i} \mu M_{f}\right) .
$$

\section{B2. $B(E 3) \mathbf{s}$}

The calculation parallels the one of the previous subsection, the only difference being that the middle term, coming from the transition operator, is $\mathcal{D}_{0, \mu}^{(3)}$. The result reads

$$
\begin{array}{r}
I_{\theta}^{(E 3)}=\frac{\left(1 \pm(-1)^{L_{i}}\right)\left(1 \pm(-1)^{L_{f}}\right)}{4} \sqrt{\frac{2 L_{i}+1}{2 L_{f}+1}} \\
\left(L_{i} 3 L_{f} \mid 000\right)\left(L_{i} 3 L_{f} \mid M_{i} \mu M_{f}\right) .
\end{array}
$$

From the $\phi$ integrals we know that non-vanishing results are obtained only in the $S \rightarrow A$ and $A \rightarrow S$ cases.

In the $S \rightarrow A$ case, $L_{i}$ is even and $L_{f}$ is odd. The first factor in the rhs of Eq. (93) has the positive sign in the place of the double sign in front of the $(-1)^{L_{i}}$ term and the negative sign in the place of the double sign in front of the $(-1)^{L_{f}}$ term, resulting in a factor of 4 in the numerator. The same factor of 4 is obtained also in the $A \rightarrow S$ case. Therefore in all cases the final result reads

$$
I_{\theta}^{(E 3)}=\sqrt{\frac{2 L_{i}+1}{2 L_{f}+1}}\left(L_{i} 3 L_{f} \mid 000\right)\left(L_{i} 3 L_{f} \mid M_{i} \mu M_{f}\right) .
$$

\section{B3. $B(E 1) \mathbf{s}$}

The calculation parallels the one of the previous subsection, the only difference being that the middle term, coming from the transition operator, is $\mathcal{D}_{0, \mu}^{(1)}$. The result reads

$$
\begin{array}{r}
I_{\theta}^{(E 1)}=\frac{\left(1 \pm(-1)^{L_{i}}\right)\left(1 \pm(-1)^{L_{f}}\right)}{4} \sqrt{\frac{2 L_{i}+1}{2 L_{f}+1}} \\
\left(L_{i} 1 L_{f} \mid 000\right)\left(L_{i} 1 L_{f} \mid M_{i} \mu M_{f}\right) .
\end{array}
$$

From the $\phi$ integrals we know that non-vanishing results are obtained only in the $S \rightarrow A$ and $A \rightarrow S$ cases.

In the $S \rightarrow A$ case, $L_{i}$ is even and $L_{f}$ is odd. The first factor in the rhs of Eq. (95) has the positive sign in the place of the double sign in front of the $(-1)^{L_{i}}$ term and the negative sign in the place of the double sign in front of the $(-1)^{L_{f}}$ term, resulting in a factor of 4 in the numerator. The same factor of 4 is obtained also in the $A \rightarrow S$ case. Therefore in all cases the final result reads

$$
I_{\theta}^{(E 1)}=\sqrt{\frac{2 L_{i}+1}{2 L_{f}+1}}\left(L_{i} 1 L_{f} \mid 000\right)\left(L_{i} 1 L_{f} \mid M_{i} \mu M_{f}\right) .
$$

\section{B4. Normalization}

Normalization of the $\theta$ wave functions is guaranteed by the integral of Eq. (4.6.1) of Ref. 66

$$
\int d^{3} \theta \mathcal{D}_{k_{1} m_{1}}^{\left(j_{1}\right) *}(\theta) \mathcal{D}_{k_{2} m_{2}}^{\left(j_{2}\right)}(\theta)=\delta_{k_{1} k_{2}} \delta_{m_{1} m_{2}} \delta_{j_{1} j_{2}} \frac{8 \pi^{2}}{2 j_{1}+1} .
$$

The normalization integral for any state reads

$$
\begin{aligned}
I_{\text {norm }}=\int d^{3} \theta \sqrt{\frac{2 L+1}{32 \pi^{2}}}\left[1 \pm(-1)^{L}\right] \mathcal{D}_{0, M}^{L *}(\theta) \\
\sqrt{\frac{2 L+1}{32 \pi^{2}}}\left[1 \pm(-1)^{L}\right] \mathcal{D}_{0, M}^{L}(\theta) .
\end{aligned}
$$

Using Eq. 97) this gives

$$
I_{\text {norm }}=\frac{1+(-1)^{2 L} \pm 2(-1)^{L}}{4}=1,
$$

since for symmetric states the positive sign appears in the place of the double sign and $L$ is even, while for antisymmetric states the negative sign appears in the place of the double sign and $L$ is odd.

\section{Appendix C. $\tilde{\beta}$ integrals}

\section{C1. $B(E 2) \mathbf{s}$}

The transition operator contains a $\tilde{\beta}$ factor, thus the integrals appearing in this case read

$$
I_{\tilde{\beta}}^{(E 2)}=\sqrt{\frac{2\left(n_{i}\right) !}{\Gamma\left(n_{i}+a_{i}+1\right)} \frac{2\left(n_{f}\right) !}{\Gamma\left(n_{f}+a_{f}+1\right)}} I^{(E 2)}\left(n_{i}, n_{f}\right),
$$

with

$$
I^{(E 2)}\left(n_{i}, n_{f}\right)=\int_{0}^{\infty} \tilde{\beta}^{a_{i}+a_{f}+2} e^{-\tilde{\beta}^{2}} L_{n_{i}}^{a_{i}}\left(\tilde{\beta}^{2}\right) L_{n_{f}}^{a_{f}}\left(\tilde{\beta}^{2}\right) d \tilde{\beta} .
$$

Using the substitution $\tilde{\beta}^{2}=x$ with $d x=2 \tilde{\beta} d \tilde{\beta}$, the integral is written as

$$
I^{(E 2)}\left(n_{i}, n_{f}\right)=\frac{1}{2} \int_{0}^{\infty} e^{-x} x^{\frac{a_{i}}{2}+\frac{a_{f}}{2}+\frac{1}{2}} L_{n_{i}}^{a_{i}}(x) L_{n_{f}}^{a_{f}}(x) d x .
$$


Analytic expressions can be found for these integrals in the case in which one of the quantum numbers $n_{i}, n_{f}$ is zero. (For the case in which both quantum numbers $n_{i}$ and $n_{f}$ are non-zero, see Eq. (B5) of Ref. 67.) We consider $n_{f}=0$, since both the ground state band and the octupole band are characterized by this value. Then one has $L_{n_{f}=0}^{a_{f}}(x)=1$ and the integral is simplified into

$$
I^{(E 2)}\left(n_{i}, 0\right)=\frac{1}{2} \int_{0}^{\infty} e^{-x} x^{\frac{a_{i}}{2}+\frac{a_{f}}{2}+\frac{1}{2}} L_{n_{i}}^{a_{i}}(x) d x .
$$

Integrals of this form are known to have the following analytic solution (68, p. 463, Eq. (5))

$$
\int_{0}^{\infty} e^{-c x} x^{(\alpha-1)} L_{n}^{\lambda}(c x) d x=\frac{(1-\alpha+\lambda)_{n}}{n ! c^{\alpha}} \Gamma(\alpha),
$$

where $(a)_{n}$ is the Pochhammer symbol

$$
(a)_{n}=\frac{\Gamma(a+n)}{\Gamma(a)} \text {. }
$$

By replacing $c=1, \lambda=a_{i}, \alpha-1=\frac{a_{i}}{2}+\frac{a_{f}}{2}+\frac{1}{2}$, i.e. $\alpha=\frac{a_{i}}{2}+\frac{a_{f}}{2}+\frac{3}{2}$, and applying the definition 105 the result is

$$
I^{(E 2)}\left(n_{i}, 0\right)=\frac{1}{2} \frac{\Gamma\left(n_{i}+\frac{a_{i}}{2}-\frac{a_{f}}{2}-\frac{1}{2}\right)}{n_{i} ! \Gamma\left(\frac{a_{i}}{2}-\frac{a_{f}}{2}-\frac{1}{2}\right)} \Gamma\left(\frac{a_{i}}{2}+\frac{a_{f}}{2}+\frac{3}{2}\right) .
$$

In the simplest case of a transition between states with $n_{i}=0$ and $n_{f}=0$, which will be eventually of major interest in the present work, one obviously has

$$
I^{(E 2)}(0,0)=\frac{1}{2} \Gamma\left(\frac{a_{i}}{2}+\frac{a_{f}}{2}+\frac{3}{2}\right) .
$$

Substituting these results in Eq. 100, for the case with $n_{i} \geq 0$ and $n_{f}=0$ we find

$$
\begin{aligned}
& I_{\tilde{\beta}}^{(E 2)}= \\
& \quad \frac{\Gamma\left(\frac{a_{i}}{2}+\frac{a_{f}}{2}+\frac{3}{2}\right) \Gamma\left(n_{i}+\frac{a_{i}}{2}-\frac{a_{f}}{2}-\frac{1}{2}\right)}{\sqrt{n_{i} ! \Gamma\left(n_{i}+a_{i}+1\right) \Gamma\left(a_{f}+1\right)} \Gamma\left(\frac{a_{i}}{2}-\frac{a_{f}}{2}-\frac{1}{2}\right)},
\end{aligned}
$$

while in the simplest case of $n_{i}=0$ and $n_{f}=0$ one has

$$
I_{\tilde{\beta}}^{(E 2)}=\frac{\Gamma\left(\frac{a_{i}}{2}+\frac{a_{f}}{2}+\frac{3}{2}\right)}{\sqrt{\Gamma\left(a_{i}+1\right) \Gamma\left(a_{f}+1\right)}} .
$$

In the case of $n_{i}=0, n_{f} \geq 0$, following the same steps one finds

$$
\begin{aligned}
& I^{(E 2)}\left(0, n_{f}\right)= \\
& \quad \frac{1}{2} \frac{\Gamma\left(n_{f}+\frac{a_{f}}{2}-\frac{a_{i}}{2}-\frac{1}{2}\right)}{n_{f} ! \Gamma\left(\frac{a_{f}}{2}-\frac{a_{i}}{2}-\frac{1}{2}\right)} \Gamma\left(\frac{a_{i}}{2}+\frac{a_{f}}{2}+\frac{3}{2}\right), \\
& I_{\tilde{\beta}}^{(E 2)}= \\
& \frac{\Gamma\left(\frac{a_{i}}{2}+\frac{a_{f}}{2}+\frac{3}{2}\right) \Gamma\left(n_{f}+\frac{a_{f}}{2}-\frac{a_{i}}{2}-\frac{1}{2}\right)}{\sqrt{n_{f} ! \Gamma\left(n_{f}+a_{f}+1\right) \Gamma\left(a_{i}+1\right)} \Gamma\left(\frac{a_{f}}{2}-\frac{a_{i}}{2}-\frac{1}{2}\right)} .
\end{aligned}
$$

\section{C2. $B(E 3) \mathbf{s}$}

The transition operator again contains a $\tilde{\beta}$ factor, thus the integrals appearing in this case are exactly the same as in the previous subsection

$$
I_{\tilde{\beta}}^{(E 3)}=I_{\tilde{\beta}}^{(E 2)} .
$$

\section{C3. $B(E 1) \mathbf{s}$}

The transition operator contains a $\tilde{\beta}^{2}$ factor, thus the integrals appearing in this case read

$$
I_{\tilde{\beta}}^{(E 1)}=\sqrt{\frac{2\left(n_{i}\right) !}{\Gamma\left(n_{i}+a_{i}+1\right)} \frac{2\left(n_{f}\right) !}{\Gamma\left(n_{f}+a_{f}+1\right)}} I^{(E 1)}\left(n_{i}, n_{f}\right),
$$

with

$$
I^{(E 1)}\left(n_{i}, n_{f}\right)=\int_{0}^{\infty} \tilde{\beta}^{a_{i}+a_{f}+3} e^{-\tilde{\beta}^{2}} L_{n_{i}}^{a_{i}}\left(\tilde{\beta}^{2}\right) L_{n_{f}}^{a_{f}}\left(\tilde{\beta}^{2}\right) d \tilde{\beta} .
$$

Using again the substitution $\tilde{\beta}^{2}=x$ with $d x=2 \tilde{\beta} d \tilde{\beta}$, the integral is written as

$$
I^{(E 1)}\left(n_{i}, n_{f}\right)=\frac{1}{2} \int_{0}^{\infty} e^{-x} x^{\frac{a_{i}}{2}+\frac{a_{f}}{2}+1} L_{n_{i}}^{a_{i}}(x) L_{n_{f}}^{a_{f}}(x) d x .
$$

For $n_{f}=0$ the integral is simplified into

$$
I^{(E 1)}\left(n_{i}, 0\right)=\frac{1}{2} \int_{0}^{\infty} e^{-x} x^{\frac{a_{i}}{2}+\frac{a_{f}}{2}+1} L_{n_{i}}^{a_{i}}(x) d x .
$$

Using Eq. 104 with $c=1, \lambda=a_{i}, \alpha-1=\frac{a_{i}}{2}+\frac{a_{f}}{2}+1$, i.e. $\alpha=\frac{a_{i}}{2}+\frac{a_{f}}{2}+2$, and applying the definition 105 the result is

$$
I^{(E 1)}\left(n_{i}, 0\right)=\frac{1}{2} \frac{\Gamma\left(n_{i}+\frac{a_{i}}{2}-\frac{a_{f}}{2}-1\right)}{n_{i} ! \Gamma\left(\frac{a_{i}}{2}-\frac{a_{f}}{2}-1\right)} \Gamma\left(\frac{a_{i}}{2}+\frac{a_{f}}{2}+2\right) .
$$

In the simplest case of $n_{i}=0$ and $n_{f}=0$ one has

$$
I^{(E 1)}(0,0)=\frac{1}{2} \Gamma\left(\frac{a_{i}}{2}+\frac{a_{f}}{2}+2\right) .
$$

Substituting these results in Eq. (113), for the case with $n_{i} \geq 0$ and $n_{f}=0$ we find

$$
\begin{aligned}
& I_{\tilde{\beta}}^{(E 1)}= \\
& \quad \frac{\Gamma\left(\frac{a_{i}}{2}+\frac{a_{f}}{2}+2\right) \Gamma\left(n_{i}+\frac{a_{i}}{2}-\frac{a_{f}}{2}-1\right)}{\sqrt{n_{i} ! \Gamma\left(n_{i}+a_{i}+1\right) \Gamma\left(a_{f}+1\right)} \Gamma\left(\frac{a_{i}}{2}-\frac{a_{f}}{2}-1\right)},
\end{aligned}
$$

while in the simplest case of $n_{i}=0$ and $n_{f}=0$ one has

$$
I_{\tilde{\beta}}^{(E 1)}=\frac{\Gamma\left(\frac{a_{i}}{2}+\frac{a_{f}}{2}+2\right)}{\sqrt{\Gamma\left(a_{i}+1\right) \Gamma\left(a_{f}+1\right)}} .
$$


In the case of $n_{i}=0, n_{f} \geq 0$, following the same steps one finds

$$
\begin{gathered}
I^{(E 1)}\left(0, n_{f}\right)=\frac{1}{2} \frac{\Gamma\left(n_{f}+\frac{a_{f}}{2}-\frac{a_{i}}{2}-1\right)}{n_{f} ! \Gamma\left(\frac{a_{f}}{2}-\frac{a_{i}}{2}-1\right)} \Gamma\left(\frac{a_{i}}{2}+\frac{a_{f}}{2}+2\right), \\
I_{\tilde{\beta}}^{(E 1)}= \\
\frac{\Gamma\left(\frac{a_{i}}{2}+\frac{a_{f}}{2}+2\right) \Gamma\left(n_{f}+\frac{a_{f}}{2}-\frac{a_{i}}{2}-1\right)}{\sqrt{n_{f} ! \Gamma\left(n_{f}+a_{f}+1\right) \Gamma\left(a_{i}+1\right)} \Gamma\left(\frac{a_{f}}{2}-\frac{a_{i}}{2}-1\right)} .
\end{gathered}
$$

\section{C4. Normalization}

The total wave functions are given in Eqs. (2) and 200. The integration over the Euler angles $\theta$ and the relevant normalization have been studied in Appendix $\mathrm{B}$, while the rest of the integrations are performed over $\iint \beta_{2}^{3} d \beta_{2} \beta_{3}^{3} d \beta_{3}$, where the $\beta_{2}^{3}, \beta_{3}^{3}$ factors come from the volume element and cancel with the first factor of Eq. (20). Using Eqs. (4) and (5), as well as the relevant Jacobian, one finds that the rest of the integrations are over $\iint d \beta_{2} d \beta_{3}=\frac{B}{\sqrt{B_{2} B_{3}}} \iint \tilde{\beta} d \tilde{\beta} d \phi$. The integration over $\phi$ and the relevant normalization factors have been studied in Appendix A. We determine here the normalization factors related to the $\tilde{\beta}$ integration. We have

$$
\frac{1}{N_{\tilde{\beta}}^{2}}=\frac{2(n) !}{\Gamma(n+a+1)} \frac{B}{\sqrt{B_{2} B_{3}}} I(n, n)
$$

with

$$
I(n, n)=\int_{0}^{\infty} \tilde{\beta}^{2 a+1} e^{-\tilde{\beta}^{2}} L_{n}^{a}\left(\tilde{\beta}^{2}\right) L_{n}^{a}\left(\tilde{\beta}^{2}\right) d \tilde{\beta} .
$$

Using the substitution $\tilde{\beta}^{2}=x$ with $d x=2 \tilde{\beta} d \tilde{\beta}$, the integral is written as

$$
I(n, n)=\frac{1}{2} \int_{0}^{\infty} e^{-x} x^{a} L_{n}^{a}(x) L_{n}^{a}(x) d x .
$$

Considering the case with $n=0$, which is of interest here, this integral is of the form of Eq. (104) with $c=1$, $\alpha-1=a$, thus leading to

$$
I(0,0)=\frac{\Gamma(a+1)}{2} .
$$

Then Eq. 123 for $n=0$ leads to

$$
\frac{1}{N_{\tilde{\beta}}^{2}}=\frac{B}{\sqrt{B_{2} B_{3}}} .
$$

This result indicates that when calculating $\tilde{\beta}$-integrals in $B(E L) \mathrm{s}$, the $N_{\tilde{\beta}}$ normalization factors cancel out with the $\frac{B}{\sqrt{B_{2} B_{3}}}$ factor appearing in the volume element and therefore do not affect the final results.

\section{Appendix D.}

\section{D1. Kinetic energy and volume elements}

The expressions for the kinetic energy and the volume element depend on the dimensionality of the space considered. We distinguish three cases, with dimensionality five, four, and three respectively.

1) In the usual Bohr Hamiltonian describing the quadrupole degree of freedom in the five-dimensional (5D) space of the collective variables $\beta$ and $\gamma$ and the three Euler angles $(\theta, \phi, \psi)$, the kinetic energy term reads 69 .

$$
\begin{aligned}
& T_{(\beta, \gamma) v i b}= \\
& -\frac{\hbar^{2}}{2 B}\left[\frac{1}{\beta^{4}} \frac{\partial}{\partial \beta} \beta^{4} \frac{\partial}{\partial \beta}+\frac{1}{\beta^{2} \sin 3 \gamma} \frac{\partial}{\partial \gamma} \sin 3 \gamma \frac{\partial}{\partial \gamma}\right],
\end{aligned}
$$

resulting from the Pauli-Podolsky quantization procedure [70] in the full 5D space. The volume element reads 69 .

$$
d \tau=\beta^{4}|\sin 3 \gamma| \sin \theta d \beta d \gamma d \theta d \phi d \psi .
$$

If the $\beta$ variable is separated from the rest, either exactly, as in the $\mathrm{E}(5)$ critical point symmetry [18, or through an adiabatic approximation, as in the $\mathrm{X}(5)$ approach [16], the volume element in the $\beta$ part of the problem becomes [16, 18 .

$$
d \tau_{\beta}(\beta)=\beta^{4} d \beta .
$$

2) In the Davydov-Chaban approach [71 the $\gamma$ variable is removed from the Hamiltonian from the very beginning of the problem, since $\gamma$ is treated as an effective deformation parameter. Then the quantization procedure is applied in the $4 \mathrm{D}$ curvilinear space of $\beta$ and the three Euler angles. As a result the kinetic energy term of the Hamiltonian is obtained in the form

$$
T_{(\beta) v i b}=-\frac{\hbar^{2}}{2 B}\left[\frac{1}{\beta^{3}} \frac{\partial}{\partial \beta} \beta^{3} \frac{\partial}{\partial \beta}\right] .
$$

Note that now the power of $\beta$ in 131 is 3 and not 4 as in the $\beta$-part of (128), while the respective volume element is

$$
d \tau_{(\beta) v i b}(\beta)=\beta^{3} d \beta .
$$

If the wave function is sought in the form 71 .

$$
\psi(\beta)=\beta^{-3 / 2} \varphi(\beta),
$$

the kinetic energy term in the Schrödinger equation for the wave function $\varphi(\beta)$ appears in the form [71]

$$
\widetilde{T}_{(\beta) v i b}=-\frac{\hbar^{2}}{2 B_{2}} \frac{\partial^{2}}{\partial \beta_{2}^{2}}+\frac{3 \hbar^{2}}{8 B_{2} \beta_{2}^{2}},
$$

where the second term is further moved into the effective potential part. 
3) In the limit of strong $\gamma$ instability of the Wilets-Jean approach [72] the nucleus is considered as a droplet which can only execute axially symmetric vibrations. This system has only three degrees of freedom: $\beta, \theta$ and $\phi$. Then the kinetic energy term in the Hamiltonian becomes

$$
T=-\frac{\hbar^{2}}{2 B} \frac{\partial^{2}}{\partial \beta^{2}},
$$

where wave functions of the form $\psi(\beta)=\beta^{-1} \varphi(\beta)$ are considered and the volume element reads

$$
d \tau=\beta^{2} \sin \theta d \beta d \theta d \phi .
$$

This approach has been recently used in Ref. [73].

The kinetic energy term of the Davydov-Chaban approach has been generalized from quadrupole to any multipolarity $\lambda$ by Williams and Davidson [74, the final result being

$$
T_{\lambda}=-\frac{\hbar^{2}}{2 B_{\lambda}}\left[\frac{1}{\beta_{\lambda}^{3}} \frac{\partial}{\partial \beta_{\lambda}} \beta_{\lambda}^{3} \frac{\partial}{\partial \beta_{\lambda}}\right] .
$$

The basic assumption behind this derivation is the requirement of no vibration-rotation cross terms [74, 75, which diagonalizes the inertial tensor and hence the rotated coordinate system is the principal inertial (body) system.

In the case of simultaneous presence of quadrupole and octupole deformation, the kinetic energy within this generalized Davydov-Chaban approach reads

$$
T_{\left(\beta_{2}, \beta_{3}\right) v i b}=-\sum_{\lambda=2,3} \frac{\hbar^{2}}{2 B_{\lambda}} \frac{1}{\beta_{\lambda}^{3}} \frac{\partial}{\partial \beta_{\lambda}} \beta_{\lambda}^{3} \frac{\partial}{\partial \beta_{\lambda}} .
$$

Using wave functions of the form

$$
\psi^{ \pm}\left(\beta_{2}, \beta_{3}\right)=\left(\beta_{2} \beta_{3}\right)^{-3 / 2} \varphi^{ \pm}\left(\beta_{2}, \beta_{3}\right),
$$

which is a straightforward generalization of Eq. 133, the kinetic energy takes the form

$$
\widetilde{T}_{\left(\beta_{2}, \beta_{3}\right) v i b}=-\sum_{\lambda=2,3}\left(\frac{\hbar^{2}}{2 B_{\lambda}} \frac{\partial^{2}}{\partial \beta_{\lambda}^{2}}+\frac{3 \hbar^{2}}{8 B_{\lambda} \beta_{\lambda}^{2}}\right),
$$

where again the second term is pushed into the effective potential, as in Eq. (134).

From the considerations given above, it becomes clear that the kinetic energy term used in Refs. [15, 51, 52, as well as in the present work, is based on the following assumptions:

1) The $\gamma$ degree of freedom is frozen from the very beginning, thus reducing the degrees of freedom to four $(\beta$, three Euler angles) in the case of pure quadrupole deformation, and to five $\left(\beta_{2}, \beta_{3}\right.$, three Euler angles) in the case of simultaneous presence of quadrupole and octupole deformations.

2) Vibration-rotation cross terms are ignored, making the inertial tensor diagonal and allowing the rotated coordinate system to be the principal inertial (body) system.

\section{D2. Moments of inertia}

Using the standard Bohr expression for the nuclear surface in the body-fixed frame, given by 69

$$
R(\theta, \phi)=R_{0}\left[1+\sum_{l m} a_{l m} Y_{l m}(\theta, \phi)\right],
$$

where $Y_{l m}(\theta, \phi)$ stands for the spherical harmonics, ignoring vibration-rotation cross terms as above, and assuming that only the even components $\left(a_{30}, a_{3 \pm 2}\right)$ of the octupole parameters are non-vanishing, we obtain for the moments of inertia in the octupole degree of freedom the expressions [75, 76]

$$
\begin{aligned}
& \mathfrak{J}_{1}^{(3)}=B_{3}\left(6 a_{30}^{2}+2 \sqrt{30} a_{30} a_{32}+8 a_{32}^{2}\right) \\
& \mathfrak{J}_{2}^{(3)}=B_{3}\left(6 a_{30}^{2}-2 \sqrt{30} a_{30} a_{32}+8 a_{32}^{2}\right) \\
& \mathfrak{J}_{3}^{(3)}=8 B_{3} a_{32}^{2},
\end{aligned}
$$

which in the axial case $\left(a_{30}=\beta_{3}, a_{32}=0\right)$ give

$$
\mathfrak{J}_{1}^{(3)}=\mathfrak{J}_{2}^{(3)}=6 B_{3} \beta_{3}^{2}, \quad \mathfrak{J}_{3}^{(3)}=0 .
$$

Different expressions for the moments of inertia are obtained if one considers the odd components $\left(a_{3 \pm 1}, a_{3 \pm 3}\right)$ as the non-vanishing ones 75 . Here we make the assumption, as in Ref. [75, 76], that for low-lying collective negative parity states the even components play the main role, since their contributions to the shape are more symmetric, a property usually associated with lower energy configurations.

For the moments of inertia in the quadrupole degree of freedom we use the standard expression 69]

$$
\mathfrak{J}_{k}^{(2)}=4 B_{2} \beta_{2}^{2} \sin ^{2}\left(\gamma-\frac{2}{3} \pi k\right),
$$

which in the axial case $(\gamma=0)$ gives

$$
\mathfrak{J}_{1}^{(2)}=\mathfrak{J}_{2}^{(2)}=3 B_{2} \beta_{2}^{2}, \quad \mathfrak{J}_{3}^{(2)}=0 .
$$

Collecting (145) and 147) into the axial quadrupoleoctupole moment of inertia one gets

$$
\mathfrak{J}_{1}^{(2+3)}=\mathfrak{J}_{2}^{(2+3)}=\mathfrak{J}^{(2+3)}=3 B_{2} \beta_{2}^{2}+6 B_{3} \beta_{3}^{2},
$$

which gives exactly the denominator in the angular momentum part of Hamiltonian (1)

$$
\frac{1}{2 \mathfrak{J}^{(2+3)}}=\frac{1}{6\left(B_{2} \beta_{2}^{2}+2 B_{3} \beta_{3}^{2}\right)} .
$$

From the considerations given above, it becomes clear that the moment of inertia term used in Refs. [15, 52, as well as in the present work, is based on the following assumptions:

1) Vibration-rotation cross terms are ignored, as in the case of the kinetic energy. 
2) Only the axial components of deformation are taken into account, both in the quadrupole and in the octupole degree of freedom, based on the qualitative expectation that more symmetric configurations would lie lower in energy.

It should be noted that in Ref. [51 an expression $3\left(B_{2} \beta_{2}^{2}+B_{3} \beta_{3}^{2}\right)$ has been used for the moment of inertia.

\section{D3. Separation of variables}

Exact separation of the $\beta$ and $\gamma$ variables in the framework of the Bohr Hamiltonian can be achieved by considering potentials of the form $u(\beta, \gamma)=v(\beta)+w(\gamma) / \beta^{2}$ [72, 77. In contrast, when the potential is of the form $u(\beta, \gamma)=v(\beta)+w(\gamma)$, only approximate adiabatic separation of variables can be tried, as in the case of the $\mathrm{X}(5)$ critical point symmetry [16, 78. In the case of $\mathrm{X}(5)$, a $\beta^{2}$ term survives in the differential equation involving the $\gamma$ variable, replaced in the adiabatic approximation by the average value $\left\langle\beta^{2}\right\rangle$. The accuracy of this approximation has been tested in Ref. [79] and the limits of its validity have been pointed out. The recently developed Algebraic Collective Model 80 83] offers a path for avoiding this approximation by performing rapidly converging exact numerical calculations instead of pursuing approximate analytical solutions.

In the present case, the $\gamma$ variable has been "frozen" from the very beginning, following the Davydov-Chaban approach [71], as explained in Appendix D1. Therefore, no question of separating the $\beta$ and $\gamma$ variables appears. However, separation of the $\beta$ and $\phi$ variables is desirable, in order to achieve analytical solutions in closed form. By analogy to the $\mathrm{X}(5)$ situation described above, a potential of the form $v(\tilde{\beta}, \phi)=u(\tilde{\beta})+w\left(\tilde{\phi}^{ \pm}\right)$has been chosen and adiabatic separation of variables has been tried, taking advantage of the fact that the $w\left(\tilde{\phi}^{ \pm}\right)$potential is supposed to be of the form of two very steep harmonic oscillators centered at the values $\pm \phi_{0}$. Because of the steepness of the oscillators it is plausible to use the adiabatic approximation in the differential equation involving the $\tilde{\beta}$ variable [Eq. (7)], by replacing the variable $\phi$ by $\pm \phi_{0}$. Again in analogy to the X(5) case mentioned above, a $\left\langle\tilde{\beta}^{2}\right\rangle$ term remains in the differential equation involving the $\tilde{\phi}$ degree of freedom [Eq. (8)]. There is no need to explicitly determine $\left\langle\tilde{\beta}^{2}\right\rangle$, since it enters the parameter $b$ [Eq. 15]], determined from $E 2$ transitions as described in subsection III.B.1.

In other words, we exploit for the separation of variables the fact that the $w\left(\tilde{\phi}^{ \pm}\right)$potential is supposed to be of the form of two very steep harmonic oscillators centered at the values $\pm \phi_{0}$. This makes the adiabatic approximation of $\phi$ by $\pm \phi_{0}$ plausible, isolating the two very steep harmonic oscillators in the $\phi$ equation and leaving the rest of the terms in the $\tilde{\beta}$ equation. An alternative possibility is to consider a potential of the form $v(\tilde{\beta}, \phi)=u(\tilde{\beta})+w\left(\tilde{\phi}^{ \pm}\right) / \tilde{\beta}^{2}$. Then the separation of variables will become exact, but the distribution of terms in the two equations will be different.

The adiabatic approximation used here, based on two very steep harmonic oscillators, does have a cost. It is well known that the correct description of the parity splitting, usually depicted as the odd-even staggering of the energy levels of the ground state band and the negative parity band, requires a finite barrier between the two wells, which is angular momentum dependent [55] 57 . In the present approach a practically infinite barrier between the two wells is used for all angular momenta. This has as a consequence that the theoretical predictions for the low lying negative parity states (especially for $1^{-}$and $3^{-}$) are poor, as pointed out in subsection III.A.

It should be mentioned that the Bohr Hamiltonian has been solved for the potential $1 / \sin ^{2}(3 \gamma)$ [resembling the last fractional term in Eq. (7)], possessing a minimum at $\gamma=\pi / 6$, first by replacing the $\gamma$ variable in the moments of inertia by its expectation value, $\gamma_{0}$, and subsequently avoiding this approximation 84 , the results revealing the approximation to be a good one. Future tests of similar nature in the present framework are desirable.

\section{D4. Comparison to other approaches}

As it has already been mentioned in the Introduction, a more general approach has been developed by Bizzeti and Bizzeti-Sona [19, 20, in which nonaxial contributions, small but not frozen to zero, are taken into account. It is worth commenting briefly on the relation between the two approaches.

1) In the AQOA approach, no nonaxial contributions are taken into account. As a result, all variables related to nonaxiality in Ref. [19] are vanishing, the matrix of inertia (Table I and II in Ref. 19) becoming diagonal.

2) Because of the same reason, in the invariants up to fourth order reported in Table VIII of Ref. [19], only the first term in each invariant, containing only $\beta_{2}$ and/or $\beta_{3}$, is surviving. In the present approach only the invariants up to second order, being equal to $\beta_{2}^{2}$ and $\beta_{3}^{2}$, are used.

3) In Ref. [19, in addition to the infinite square well potential, a harmonic oscillator potential proportional to the square of $x=\sqrt{2} \beta_{3} / \beta_{2}$ has been used. In the present approach, $\beta_{3} / \beta_{2}=\tan \phi \sqrt{B_{2} / B_{3}}$. Therefore the two potentials coincide, up to constant factors, for small angles, for which $\tan \phi \approx \sin \phi \approx \phi$.

4) The total moment of inertia appearing in Ref. 19 [Eq. (32)], coincides with the total moment of inertia used here [Eq. 149p], if the nonaxial variables vanish, as seen from Eq. (6a) of Ref. 19.
[1] A. Bohr and B. R. Mottelson, Nuclear Structure, Vol. II (Benjamin, New York, 1975).
[2] S. G. Rohoziński, Octupole vibrations in nuclei, Rep. 
Prog. Phys. 51, 541 (1988).

[3] I. Ahmad and P. A. Butler, Octupole shapes in nuclei, Annu. Rev. Nucl. Part. Sci. 43, 71 (1993).

[4] P. A. Butler and W. Nazarewicz, Intrinsic reflection asymmetry in atomic nuclei, Rev. Mod. Phys. 68, 349 (1996).

[5] P. Schüler, Ch. Lauterbach, Y. K. Agarwal, J. De Boer, K. P. Blume, P. A. Butler, K. Euler, Ch. Fleischmann, C. Günther, E. Hauber, H. J. Maier, M. Marten-Tölle, Ch. Schandera, R. S. Simon, R. Tölle, and P. Zeyen, High-spin states in ${ }^{224,226,228} \mathrm{Th}$ and the systematics of octupole effects in even Th isotopes, Phys. Lett. B 174, 241 (1986).

[6] I. Wiedenhöver, R. V. F. Jannsens, G. Hackman, I. Ahmad, J. P. Greene, H. Amro, P. K. Bhattacharyya, M. P. Carpenter, P. Chowdhury, J. Cizewski, D. Cline, T. L. Khoo, T. Lauritsen, C. J. Lister, A. O. Macchiavelli, D. T. Nisius, P. Reiter, E. H. Seabury, D. Seweryniak, S. Siem, A. Sonzogni, J. Uusitalo, and C. Y. Wu, Octupole correlations in the $\mathrm{Pu}$ isotopes: From vibration to static deformation?, Phys. Rev. Lett. 83, 2143 (1999).

[7] J. F. C. Cocks, P. A. Butler, K. J. Cann, P. T. Greenlees, G. D. Jones, S. Asztalos, P. Bhattacharyya, R. Broda, R. M. Clark, M. A. Deleplanque, R. M. Diamond, P. Fallon, B. Fornal, P. M. Jones, R. Julin, T. Lauritsen, I. Y. Lee, A. O. Macchiavelli, R. W. MacLeod, J. F. Smith, F. S. Stephens, and C. T. Zhang, Observation of octupole structures in radon and radium isotopes and their contrasting behavior at high spin, Phys. Rev. Lett. 78, 2920 (1997).

[8] J. F. C. Cocks, D. Hawcroft, N. Amzal, P. A. Butler, K. J. Cann, P. T. Greenlees, G. D. Jones, S. Asztalos, R. M. Clark, M. A. Deleplanque, R. M. Diamond, P. Fallon, I. Y. Lee, A. O. Macchiavelli, R. W. MacLeod, F. S. Stephens, P. Jones, R. Julin, R. Broda, B. Fornal, J. F. Smith, T. Lauritsen, P. Bhattacharyya, and C. T. Zhang, Spectroscopy of Rn, Ra and Th isotopes using multi-nucleon transfer reactions, Nucl. Phys. A 645, 61 (1999).

[9] W. R. Phillips, I. Ahmad, H. Emling, R. Holzmann, R. V. F. Janssens, T. L. Khoo, and M. W. Drigert, Octupole deformation in neutron-rich barium isotopes, Phys. Rev. Lett. 57, 3257 (1986).

[10] W. R. Phillips, R. V. F. Janssens, I. Ahmad, H. Emling, R. Holzmann, T. L. Khoo, and M. W. Drigert, Octupole correlation effects near $Z=56, N=88$, Phys. Lett. B 212, 402 (1988).

[11] P. D. Cottle, Possible static octupole deformation at high angular momentum in ${ }^{78} \mathrm{Kr}$ and ${ }^{126,128} \mathrm{Ba}$, Phys. Rev. C 41, 517 (1990).

[12] W. Nazarewicz, P. Olanders, I. Ragnarsson, J. Dudek, G. A. Leander, P. Möller, and E. Ruchowska, Analysis of octupole instability in medium-mass and heavy nuclei, Nucl. Phys. A 429, 269 (1984).

[13] W. Nazarewicz and P. Olanders, Rotational consequences of stable octupole deformation in nuclei, Nucl. Phys. A 441, 420 (1985).

[14] R. K. Sheline, Definition of the actinide region of static quadrupole-octupole deformation, Phys. Lett. B 197, 500 (1987).

[15] D. Bonatsos, D. Lenis, N. Minkov, D. Petrellis, and P. Yotov, Description of critical point actinides in a transition from octupole deformation to octupole vibrations, Phys. Rev. C 71, 064309 (2005).
[16] F. Iachello, Analytic description of critical point nuclei in a spherical-axially deformed shape phase transition, Phys. Rev. Lett. 87, 052502 (2001).

[17] P. Cejnar, J. Jolie, and R. F. Casten, Quantum phase transitions in the shapes of atomic nuclei, Rev. Mod. Phys. 82, 2155 (2010).

[18] F. Iachello, Dynamic symmetries at the critical point, Phys. Rev. Lett. 85, 3580 (2000).

[19] P. G. Bizzeti and A. M. Bizzeti-Sona, Description of nuclear octupole and quadrupole deformation close to the axial symmetry and phase transitions in the octupole mode, Phys. Rev. C 70, 064319 (2004).

[20] P. G. Bizzeti and A. M. Bizzeti-Sona, Description of nuclear octupole and quadrupole deformation close to axial symmetry: Critical-point behavior of ${ }^{224} \mathrm{Ra}$ and ${ }^{224} \mathrm{Th}$, Phys. Rev. C 77, 024320 (2008).

[21] L. P. Gaffney, et al., Studies of pear-shaped nuclei using accelerated radioactive beams, Nature (London) 497, 199 (2013).

[22] P. M. Davidson, Eigenfunctions for calculating electronic vibrational intensities, Proc. R. Soc. London Ser. A 135, 459 (1932).

[23] D. Bonatsos, E. A. McCutchan, N. Minkov, R. F. Casten, P. Yotov, D. Lenis, D. Petrellis, I. Yigitoglu, Separable version of the Bohr Hamiltonian with the Davidson potential, Phys. Rev. C 76, 064312 (2007).

[24] A. Artna-Cohen, Nuclear Data Sheets for A = 224, Nucl. Data Sheets 80, 227 (1997).

[25] Y. A. Akovali, Nuclear Data Sheets for A $=226$, Nucl. Data Sheets 77, 433 (1996).

[26] P. G. Bizzeti and A. M. Bizzeti-Sona, Octupole transitions in the critical-point nucleus ${ }^{224}$ Ra, Phys. Rev. C 88, 011305(R) (2013).

[27] J. Engel and F. Iachello, Quantization of asymmetric shapes in nuclei, Phys. Rev. Lett. 54, 1126 (1985).

[28] J. Engel and F. Iachello, Interacting Boson Model of collective octupole states (I). The rotational limit, Nucl. Phys. A 472, 61 (1987).

[29] D. Kusnezov, The U(16) algebraic lattice, J. Phys. A: Math. Gen. 22, 4271 (1989).

[30] D. Kusnezov, The U(16) algebraic lattice: II. analytic construction. J. Phys. A: Math. Gen. 23, 5673 (1990).

[31] N. V. Zamfir and D. Kusnezov, Octupole correlations in the transitional actinides and the sspdf interacting boson model, Phys. Rev. C 63, 054306 (2001).

[32] N. V. Zamfir and D. Kusnezov, Octupole correlations in U and Pu nuclei, Phys. Rev. C 67, 014305 (2003).

[33] S. Kuyucak, Shape-phase transitions in mixed parity systems and the onset of octupole deformation, Phys. Lett. B 466, 79 (1999).

[34] S. Kuyucak and M. Honma, Mean field study of the quadrupole-octupole degree of freedom in the spdf boson model, Phys. Rev. C 65, 064323 (2002).

[35] H. J. Daley and F. Iachello, Nuclear Vibron Model. I. The SU(3) limit, Ann. Phys. (N.Y.) 167, 73 (1986).

[36] B. Buck, A. C. Merchant, and S. M. Perez, Alternative view of collective bands in actinide nuclei, Phys. Rev. C 57, R2095 (1998).

[37] B. Buck, A. C. Merchant, and S. M. Perez, Negative parity bands in even-even isotopes of $\mathrm{Ra}$, Th, $\mathrm{U}$ and $\mathrm{Pu}$, J. Phys. G: Nucl. Part. Phys. 35, 085101 (2008).

[38] T. M. Shneidman, G. G. Adamian, N. V. Antonenko, R. V. Jolos, and W. Scheid, Cluster interpretation of properties of alternating parity bands in heavy nuclei, 
Phys. Rev. C 67, 014313 (2003).

[39] T. M. Shneidman, G. G. Adamian, N. V. Antonenko, and R. V. Jolos, Possible alternative parity bands in the heaviest nuclei, Phys. Rev. C 74, 034316 (2006).

[40] L. S. Geng, J. Meng, and T. Hiroshi, Reflection asymmetric relativistic mean field approach and its application to the octupole deformed nucleus ${ }^{226} \mathrm{Ra}$, Chin. Phys. Lett. 24, 1865 (2007).

[41] J. Y. Guo, P. Jiao, and X. Z. Fang, Microscopic description of nuclear shape evolution from spherical to octupole-deformed shapes in relativistic mean-field theory, Phys. Rev. C 82, 047301 (2010).

[42] W. Zhang, Z. P. Li, S. Q. Zhang, and J. Meng, Octupole degree of freedom for the critical-point candidate nucleus ${ }^{152} \mathrm{Sm}$ in a reflection-asymmetric relativistic mean-field approach, Phys. Rev. C 81, 034302 (2010).

[43] W. Zhang, Z. P. Li, and S. Q. Zhang, Octupole deformation for $\mathrm{Ba}$ isotopes in a reflection-asymmetric relativistic mean-field approach, Chin. Phys. C 34, 1094 (2010).

[44] K. Nomura, N. Shimizu, and T. Otsuka, Mean-field derivation of the interacting boson model Hamiltonian and exotic nuclei, Phys. Rev. Lett. 101, 142501 (2008).

[45] K. Nomura, D. Vretenar, and B. N. Lu, Microscopic analysis of the octupole phase transition in Th isotopes, Phys. Rev. C 88, 021303(R) (2013).

[46] K. Nomura, D. Vretenar, T. Nikšić, and B. N. Lu, Microscopic description of octupole shape-phase transitions in light actinides and rare-earth nuclei, Phys. Rev. C 89, 024312 (2014).

[47] A. A. Raduta, A. Faessler, and R. K. Sheline, Phenomenological description of the $K^{\pi}=0^{-}$bands in some Rn and Ra isotopes, Phys. Rev. C 57, 1512 (1998).

[48] A. A. Raduta, Al. H. Raduta, and A. Faessler, Phenomenological description of rotational bands in the pear shaped nuclei, Phys. Rev. C 55, 1747 (1997).

[49] A. A. Raduta, D. Ionescu, I. I. Ursu, and A. Faessler, New features of positive and negative parity rotational bands in ${ }^{226}$ Ra, Nucl. Phys. A 720, 43 (2003).

[50] A. A. Raduta and D. Ionescu, New signatures for octupole deformation in some actinide nuclei, Phys. Rev. C 67, 044312 (2003).

[51] A. Ya. Dzyublik and V. Yu. Denisov, Collective states of even-even nuclei with quadrupole and octupole deformations, Yad. Fiz. 56, 30 (1993) [Phys. At. Nucl. 56, 303 (1993)].

[52] V. Yu. Denisov and A. Ya. Dzyublik, Collective states of even-even and odd nuclei with $\beta_{2}, \beta_{3}, \ldots, \beta_{N}$ deformations, Nucl. Phys. A 589, 17 (1995).

[53] J. P. Elliott, J. A. Evans, and P. Park, A soluble $\gamma-$ unstable Hamiltonian, Phys. Lett. B 169, 309 (1986).

[54] D. J. Rowe and C. Bahri, Rotation-vibrational spectra of diatomic molecules and nuclei with Davidson interactions, J. Phys. A 31, 4947 (1998).

[55] R. V. Jolos and P. von Brentano, Angular momentum dependence of the parity splitting in nuclei with octupole correlations, Phys. Rev. C 49, R2301 (1994).

[56] R. V. Jolos and P. von Brentano, Rotational spectra and parity splitting in nuclei with strong octupole correlations, Nucl. Phys. A 587, 377 (1995).

[57] R. V. Jolos and P. von Brentano, Parity splitting in the alternating parity bands of some actinide nuclei, Phys. Rev. C 60, 064317 (1999).

[58] K. Heyde, Basic Ideas and Concepts in Nuclear Physics (IOP Publishing, Bristol, 1994).
[59] D. Lenis and D. Bonatsos, Parameter-free solution of the Bohr Hamiltonian for actinides critical in the pctupole mode, Phys. Lett. B 633, 474 (2006).

[60] R. V. Jolos, P. von Brentano, and J. Jolie, Second order phase transitions from octupole-nondeformed to octupole-deformed shape in the alternating parity bands of nuclei around ${ }^{240} \mathrm{Pu}$ based on data, Phys. Rev. C 86, 024319 (2012).

[61] R. V. Jolos, P. von Brentano, and R. F. Casten, Anharmonicity of the excited octupole band in actinides using supersymmetric quantum mechanics, Phys. Rev. C 88, 034306 (2013).

[62] P. G. Bizzeti and A. M. Bizzeti-Sona, Description of nuclear octupole and quadrupole deformation close to axial symmetry: Octupole vibrations in the $\mathrm{X}(5)$ nuclei ${ }^{150} \mathrm{Nd}$ and ${ }^{152}$ Sm, Phys. Rev. C 81, 034320 (2010).

[63] R. Rodríguez-Guzmán, L. M. Robledo, and P. Sarriguren, Microscopic description of quadrupole-octupole coupling in $\mathrm{Sm}$ and $\mathrm{Gd}$ isotopes with the Gogny energy density functional, Phys. Rev. C 86, 034336 (2012).

[64] M. Sugawara and H. Kusakari, X(5) and analytic quadrupole and octupole axially symmetric models applied to ${ }^{148}$ Nd, Phys. Rev. C 35, 067302 (2007).

[65] I. S. Gradshteyn and I. M. Ryzhik, Table of Integrals, Series, and Products (Academic, New York, 1980).

[66] A. R. Edmonds, Angular Momentum in Quantum Mechanics (Princeton U. Press, Princeton, 1957).

[67] N. Minkov, S. Drenska, M. Strecker, W. Scheid, and H. Lenske, Non-yrast nuclear spectra in a model of coherent quadrupole-octupole motion, Phys. Rev. C 85, 034306 (2012).

[68] A. P. Prudnikov, Yu. A. Brichkov and O. I. Marichev, Integrals and Series of Special Functions (Nauka, Moskva, 1985) (in Russian).

[69] A. Bohr, The coupling of nuclear surface oscillations to the motion of individual nucleons, Mat. Fys. Medd. K. Dan. Vidensk. Selsk. 26, no. 14 (1952).

[70] B. Podolsky, Quantum-mechanically correct form of Hamiltonian function for conservative systems, Phys. Rev. 32, 812 (1928).

[71] A. S. Davydov and A. A. Chaban, Rotation-vibration interaction in non-axial even nuclei, Nucl. Phys. 20, 499 (1960).

[72] L. Wilets and M. Jean, Surface oscillations in even-even nuclei, Phys. Rev. 102, 788 (1956).

[73] Sh. Shapirov and M. S. Nadirbekov, About collective excited states even-even nuclei with quadrupole and octupole deformations, J. Nucl. Rad. Phys. 3, 63 (2008).

[74] S. A. Williams and J. P. Davidson, A generalized rotation-vibration model for deformed even nuclei, Can. J. Phys. 40, 1423 (1962).

[75] P. O. Lipas and J. P. Davidson, Octupole vibrations of deformed even nuclei, Nucl. Phys. 26, 80 (1961).

[76] J. P. Davidson, Collective Models of the Nucleus (Academic, New York, 1968).

[77] L. Fortunato, Solutions of the Bohr Hamiltonian, a compendium, Eur. Phys. J. A 26, 1 (2005).

[78] R. Bijker, R. F. Casten, N. V. Zamfir, and E. A. McCutchan, Test of X(5) for the $\gamma$ degree of freedom, Phys. Rev. C68, 064304 (2003).

[79] M. A. Caprio, Effects of $\beta-\gamma$ coupling in transitional nuclei and the validity of the approximate separation of variables, Phys. Rev. C 72, 054323 (2005).

[80] D. J. Rowe, A computationally tractable version of the 
collective model, Nucl. Phys. A 735, 372 (2004).

[81] D. J. Rowe and P. S. Turner, The algebraic collective model, Nucl. Phys. A 753, 94 (2005).

[82] M. A. Caprio, Phonon and multi-phonon excitations in rotational nuclei by exact diagonalization of the Bohr Hamiltonian, Phys. Lett. B 672, 396 (2009).

[83] D. J. Rowe, T. A. Welsh, and M. A. Caprio, Bohr model as an algebraic collective model, Phys. Rev. C 79, 054304 (2009).

[84] S. De Baerdemacker, L. Fortunato, V. Hellemans, and K. Heyde, Solution of the Bohr Hamiltonian for a periodic potential with minimum at $\gamma=\pi / 6$, Nucl. Phys. A 769, $16(2006)$. 